\title{
Infrared Galaxies in the Field of the Massive Cluster Abell S1063: Discovery of a Luminous Kiloparsec-sized H II Region in a Gravitationally Lensed Infrared-luminous Galaxy at $z=0.6$
}

\author{
Gregory L. Walth ${ }^{1,2,3}$ (1) , Eiichi Egami ${ }^{3}$, Benjamin Clément ${ }^{4}$, Timothy D. Rawle ${ }^{5}$, Marie Rex ${ }^{3}$, Johan Richard $^{4}$ (i), \\ Pablo Pérez-González ${ }^{6,7}$, Frédéric Boone ${ }^{8}$, Miroslava Dessauges-Zavadsky ${ }^{9}$ (i), Jeff Portouw $^{3}$, Benjamin Weiner ${ }^{3}$ (1), \\ Ian McGreer $^{3}$ iD, and Evan Schneider ${ }^{10}$ (D) \\ ${ }^{1}$ The Observatories of the Carnegie Institution for Science, 813 Santa Barbara Street, Pasadena, CA 91101, USA; gwalth@carnegiescience.edu \\ ${ }^{2}$ UC San Diego, Center for Astrophysics \& Space Sciences, 9500 Gilman Drive, La Jolla, CA, USA \\ ${ }^{3}$ Steward Observatory, University of Arizona, 933 N. Cherry Avenue, Tucson, AZ 85721, USA \\ ${ }^{4}$ Univ Lyon, Univ Lyon1, Ens de Lyon, CNRS, Centre de Recherche Astrophysique de Lyon UMR5574, F-69230 Saint-Genis-Laval, France \\ ${ }^{5}$ ESA/Space Telescope Science Institute (STScI), 3700 San Martin Drive, Baltimore, MD 21218, USA \\ ${ }_{7}^{6}$ Departamento de Astrofísica, Facultad de CC. Físicas, Universidad Complutense de Madrid, E-28040 Madrid, Spain \\ ${ }^{7}$ Centro de Astrobiología (CAB, INTA-CSIC), Carretera de Ajalvir km 4, E-28850 Torrejón de Ardoz, Madrid, Spain \\ ${ }^{8}$ Université de Toulouse, UPS-OMP, CNRS, IRAP, 9 Av. colonel Roche, BP 44346, F-31028 Toulouse Cedex 4, France \\ 9 Observatoire de Genève, Université de Genève, 51 Ch. des Maillettes, CH-1290 Sauverny, Switzerland \\ ${ }^{10}$ Department of Astrophysical Sciences, Princeton University, 4 Ivy Lane, Princeton, NJ 08544, USA \\ Received 2018 January 5; revised 2019 March 19; accepted 2019 April 5; published 2019 May 16
}

\begin{abstract}
Using the Spitzer Space Telescope and Herschel Space Observatory, we have conducted a survey of IR galaxies in the field of the galaxy cluster AS1063 at $z=0.347$, which is one of the most massive clusters known and a target of the Hubble Space Telescope (HST) Cluster Lensing and Supernova Survey with Hubble and the Frontier Field surveys. The Spitzer/MIPS $24 \mu \mathrm{m}$ and Herschel/PACS and SPIRE images revealed that the core of AS1063 is surprisingly devoid of IR sources, showing only a few detectable sources within the central $r \sim 1^{\prime}$. There is, however, one particularly bright source $(2.3 \mathrm{mJy}$ at $24 \mu \mathrm{m} ; 106 \mathrm{mJy}$ at $160 \mu \mathrm{m})$, which corresponds to a background galaxy at $z=0.61$. The modest magnification factor $(4.0 \times)$ implies that this galaxy is intrinsically IR luminous $\left(L_{\mathrm{IR}}=3.1 \times 10^{11} \mathrm{~L}_{\odot}\right)$. What is particularly interesting about this galaxy is that $H S T$ optical/near-IR images show a remarkably bright and large $(1 \mathrm{kpc})$ clump at one edge of the disk. Our follow-up optical/near-IR spectroscopy shows Balmer $(\mathrm{H} \alpha-\mathrm{H} 8)$ and forbidden emission from this clump ([O II] $\lambda 3727$, [O III] $\lambda \lambda 4959,5007$, [N II] $\lambda \lambda 6548,6583)$, indicating that it is an $\mathrm{H}$ II region. The H II region appears to have formed in situ, as kinematically it is part of a rotating disk, and there is no evidence of nearby interacting galaxies. With an extinction correction of $A_{\mathrm{V}}=1.5 \mathrm{mag}$, the star formation rate of this giant $\mathrm{H}$ II region is $\sim 10 \mathrm{M}_{\odot} \mathrm{yr}^{-1}$, which is exceptionally large, even for high-redshift $\mathrm{H}$ II regions. Such a large and luminous $\mathrm{H}$ II region is often seen at $z \sim 2$ but is quite rare in the nearby universe.
\end{abstract}

Key words: galaxies: clusters: individual (AS1063) - galaxies: evolution - galaxies: formation - galaxies: kinematics and dynamics - infrared: galaxies

\section{Introduction}

Star-forming galaxies at $z \sim 2$ have clumpy morphologies (Cowie et al. 1995; Elmegreen et al. 2004a, 2004b; Förster Schreiber et al. 2011a, 2011b) and form stars more vigorously than is seen in the local universe. This vigorous mode of star formation is found in the form of "clumpy" giant kiloparsecsized H II regions. Star formation for galaxies at these redshifts occurs throughout their disks at higher rates than their local analogs. There is evidence that in the local universe these clumps form from the interaction of galaxies. A canonical picture of this interaction can be seen in the Antennae galaxies, NGC 4038/39 (Arp 244), where star clusters form where the material (gas and dust) from both galaxies collide (Schweizer 1987; Barnes \& Hernquist 1991; Holtzman et al. 1992; Whitmore et al. 1993; Mihos \& Hernquist 1996). However, at high redshift $(z>2)$, clumps like these form in isolation, which is thought to be due to them being gravitationally unstable and quickly collapsing (Bournaud et al. 2007; Dekel et al. 2009; Genzel et al. 2011).

Currently there are two ideas about the formation of these large luminous clumps: (1) they are created with the same physical process, at both low redshift and high redshift, and they are just scaled-up H II regions, in which their luminosity scales with radius, velocity dispersion, and $M_{\text {Jeans }}$ (Wisnioski et al. 2012); or (2) the high-redshift clumps are undergoing a different mode of star formation, in which global galaxy properties, such as gas fraction, give rise to the formation of larger, more luminous clumps, suggesting that they evolve with redshift (Livermore et al. 2012, 2015). It might be expected that the physical properties of the clumps evolve with redshift, because the global star formation rate (SFR) of the universe has decreased since the peak at $z \sim 2$ (Madau et al. 1998). There is some evidence of this seen in the local universe in which large luminous star-forming regions are quite rare. Galaxies in the early universe were more gas rich, leading to greater star formation; as cool dense gas becomes less available, it might be expected that the star formation would decrease along with the size of these regions. Guo et al. (2015) found that in the redshift range of $0.5<z<2$ the clump fraction has decreased over time for higher stellar mass galaxies, while the lower stellar mass galaxies' clump fraction remains almost constant. In addition, it is suggested that clumps are short-lived: either 
clumps migrate toward the bulge of a galaxy or diffuse within 0.1-1 Gyr (Dekel et al. 2009; Genzel et al. 2011; Guo et al. 2012). Conditions at higher redshift, such as higher gas fractions and cold flow accretion, enabled the regular formation of these clumps.

Field surveys of galaxies at redshift $z \sim 2$ with spatially resolved $\mathrm{H}$ II regions only probe the largest star-forming regions, typically kiloparsecs in size (Förster Schreiber et al. 2011a, 2011b). However, this may not necessarily be a reflection of "normal" star-forming regions. There may be many more star-forming regions unresolved to field surveys, which may better reflect the distribution of normal star-forming regions. In addition, with the evolution of clump size and luminosity decreasing through time, it makes it more difficult to detect and characterize intermediate-redshift clumps, even with Hubble Space Telescope (HST) resolution. In the cases where large clumps are detected, it is unclear whether they truly are a large clump or some combination of smaller unresolved clumps.

With gravitational lensing it is possible to probe subkiloparsec scales of high-redshift galaxies, taking advantage of the image of the galaxy being magnified and stretched, resolving galaxies at much higher spatial resolution, and enabling the detailed study of star-forming regions (Jones et al. 2010b; Frye et al. 2012; Livermore et al. 2012; Wuyts et al. 2014). Gravitational lensing also enables the detection of lower luminosity clumps, where the flux from relatively faint galaxies is amplified by the lens. With these two characteristics, it is possible to detect fainter clumps at subkiloparsec scales, even deblending larger clumps into several smaller clumps (Johnson et al. 2017a, 2017b; Rigby et al. 2017; Cava et al. 2018).

We have seen in the local universe that star clusters found in star-forming galaxies are bright in the ultraviolet (UV) (Meurer et al. 1995) and far-infrared (far-IR) (Armus et al. 1990). The far-IR emission is the reradiation of UV light from young stars that is scattered and absorbed by dust. Star-forming regions at high redshift have been primarily studied in the rest-frame UV due to the availability of high-resolution instrumentation in the optical and near-IR. Far-IR observations are challenging due to the sensitivity, resolution, and access to currently available instrumentation and facilities. Only a limited number of galaxies with clumps at high redshift have been studied in the far-IR. Wisnioski et al. (2013) investigated the dust properties of $13 \mathrm{UV}$ selected galaxies at $z=1.3$, from the WiggleZ sample, and found that only three were detected with Herschel. The remaining 10 were nondetections, below the sensitivity of Herschel. Currently, with ALMA, Hodge et al. (2016) could detect only the dusty disks of galaxies at $z \sim 2.5$, probing down to kiloparsec scales. Even utilizing the longest baselines of ALMA will only have comparable resolution to $H S T$, which is not enough to probe subkiloparsec scales without the aid of gravitational lensing.

In order to overcome the difficulty of detecting individual star-forming clumps at high redshift in the far-IR/submillimeter we use a gravitationally lensed sample selected with Herschel. The Herschel Lensing Survey (HLS; Egami et al. 2010) is a survey of massive galaxy clusters in the far-IR/ submillimeter using Herschel to detect gravitationally lensed galaxies in the submillimeter. HLS consists of two surveys, a deep survey (HLS-deep; $290 \mathrm{hr}$ ) of 54 clusters utilizing PACS $(100,160 \mu \mathrm{m})$ and SPIRE $(250,350,500 \mu \mathrm{m})$ and a snapshot survey (HLS-snapshot; $52 \mathrm{hr}$ ) of 527 clusters with SPIRE-only bands. One of the main goals of HLS is to identify and follow up bright gravitationally lensed galaxies near the centers of clusters. It is expected that there are very few star-forming and post-starburst galaxies that are cluster members near the projected center of the cluster. Cluster cores at low redshift are dominated by passive galaxies. The main assumption is that the majority of sources emitting in the far-IR near the cluster center are being gravitationally lensed. It would be rare to find cluster galaxies emitting in the far-IR near the cluster core, with a notable exception of brightest cluster galaxies in cool-core clusters (Rawle et al. 2012) and merging clusters, such as Abell 2744 (Rawle et al. 2014).

Abell S1063 (AS1063, RXJ2248-4431) is a particularly interesting cluster in our sample because it is one of the brightest and most massive galaxy clusters known, with an X-ray luminosity of $L_{\mathrm{X}}=(43.2 \pm 0.6) \times 10^{44} \mathrm{erg} \mathrm{s}^{-1}$ (Maughan et al. 2008) and mass $M_{200}=33.1_{-6.8}^{+9.6} \times 10^{14} \mathrm{M}_{\odot}$ (Williamson et al. 2011; Gómez et al. 2012; Gruen et al. 2013). The X-ray emission (Maughan et al. 2008) and Sunyaev-Zel'dovich signal (Williamson et al. 2011) imply that AS1063 is a relaxed cluster, or virialized. However, dynamical cluster modeling (Gómez et al. 2012) has shown that it has undergone a recent merger, which is further supported by the weak lensing analysis by Gruen et al. (2013). AS1063's mass and recent merger history make it an exciting candidate for discovering strongly lensed high-redshift galaxies, such as a quadruply imaged galaxy at redshift $z=6.1$, (Balestra et al. 2013; Boone et al. 2013; Monna et al. 2014), and studying their nebular emission (Mainali et al. 2017). Massive clusters with ongoing merging often exhibit large expanded critical lines, increasing the area in which lensed galaxies can be discovered. Gruen et al. (2013) has also shown evidence for a possible background cluster at $z \sim 0.6$, which has the potential to be an optimal lensing configuration for finding highly magnified galaxies due to the chance alignment of two mass concentrations along the line of sight (Wong et al. 2012). AS1063 is one of six Hubble Frontier Field clusters, in which deep HST Advanced Camera for Surveys (ACS) and Wide Field Camera 3 (WFC3) imaging have recently been completed with the goal of finding the highest redshift galaxies and characterizing the populations of galaxies at redshifts $z=5-10$. This type of study is beneficial for Herschel-detected galaxies, as their dusty nature obscures their $\mathrm{UV} /$ optical emission and greater UV/optical depth is necessary to detect them. Within all six Hubble Frontier Field clusters, HLS finds $~ 260 \mathrm{Herschel-detected} \mathrm{galaxies} \mathrm{with} \mathrm{an} \mathrm{optical/near-IR}$ counterpart (Rawle et al. 2016).

In this paper, we report the discovery of three IR-bright sources in the core of AS1063. Particularly interesting is the discovery of a luminous kiloparsec-sized star-forming region in one of these sources, which is a cluster-lensed IR luminous galaxy at $z=0.6$ (AS1063a). This star-forming region, showing up prominently in the $H S T$ optical/near-IR images, is similar in size and luminosity to clumpy star-forming regions found at higher redshift $(z \sim 2)$, making this galaxy an excellent lower-redshift laboratory for studying giant starforming regions at $z \sim 2$. In addition, the high spatial resolution resulting from lensing magnification allows us to study in detail the nebular emission properties of this galaxy and how they relate to the dust and gas.

The paper is outlined as follows. In Section 2 we present the sample, observations, and data reduction methods. In Section 3 we present the results and measurements of the data. In Section 4 we discuss the lens model, the physical properties of 
the galaxy, and the giant luminous star-forming region, and in Section 5 we summarize our results.

The cosmology used throughout this paper is $H_{0}=$ $70 \mathrm{~km} \mathrm{~s}^{-1} \mathrm{Mpc}^{-1}, \Omega_{\mathrm{M}}=0.3$, and $\Omega_{\Lambda}=0.7$. All magnitudes are in $\mathrm{AB}$ magnitudes, and all flux densities are in milliJanskys.

\section{Observations and Data Reduction}

\subsection{Sample}

The lensed galaxy in this paper comes from HLS-deep (Egami et al. 2010), a sample of 54 massive galaxy clusters imaged with the Herschel Space Observatory (Pilbratt et al. 2010) using PACS $(100,160 \mu \mathrm{m})$ and SPIRE (250, 350, and $500 \mu \mathrm{m})$. The galaxy clusters are selected by X-ray luminosity, which is a proxy for mass. With these massive galaxy clusters it is possible to take advantage of their lensing power to detect faint high-redshift galaxies (Rex et al. 2010; Combes et al. 2012).

\subsection{Spitzer Imaging and Spectroscopy}

Imaging for AS1063 was obtained (PI: Rieke) at 3.6, 4.5, 5.8, and $8.0 \mu \mathrm{m}$ using the Infrared Array Camera (IRAC; Fazio et al. 2004) on the Spitzer Space Telescope (Werner et al. 2004). Each channel on IRAC has a field of view (FOV) of $5 ! 2 \times 5 ! 2$ and pixel size of $\sim 1$ !' 2 pixel $^{-1}$. The images had a small dither pattern and were mosaicked together to have a final pixel scale of 0 ". 6 pixel $^{-1}$. The integration time was $2400 \mathrm{~s}$. Additional imaging for AS1063 was also obtained during the warm mission, which is part of the IRAC Lensing Survey (PI: Egami), adding to the depth of channels 1 and 2 (3.6 and $4.5 \mu \mathrm{m})$ with an integration time of $18,000 \mathrm{~s}$. The total integration times for channels 1 and 2 with the warm and cold missions combined are 20,400 s.

AS1063 is observed with Multiband Imaging Photometer for Spitzer (MIPS; Rieke et al. 2004) at $24 \mu \mathrm{m}$. MIPS has an FOV of $5^{\prime} \times 5^{\prime}$ and a pixel scale of 2 ". 45 pixel $^{-1}$. The observations were part of a program to image the fields of clusters in the mid-IR (PI: Rieke), the greatest depth covering $6^{\prime} \times 6^{\prime}$ of the cluster center with a total integration time of $3600 \mathrm{~s}$. Three bright sources were identified immediately near the cluster core (Figure 1, top right panel) and were targeted for follow-up. Photometry for both IRAC and MIPS was measured using SEXTRACTOR (Bertin \& Arnouts 1996) using the parameters FLUX_AUTO and FLUXERR_AUTO.

An InfraRed Spectrograph (IRS; Houck et al. 2004) spectrum was taken for the brightest Spitzer/MIPS $24 \mu \mathrm{m}$ source, AS1063a $(2.5 \mathrm{mJy})$, in the cluster core in Long-Low mode $(14-40 \mu \mathrm{m})$ with an integration of $2400 \mathrm{~s}$. IRS in LongLow mode has a resolution of $R=57-126$ and pixel scale of 5 !" 1 pixel $^{-1}$ along the slit. The first and second orders of LongLow mode have slit widths of 10 . 7 and 10 ."5 respectively. Line fluxes in the IRS spectrum were measured using PAHFIT (Smith et al. 2007).

\subsection{HST Imaging}

We used the publicly available HST imaging of AS1063 from the Cluster Lensing and Supernova Survey with Hubble (CLASH; Postman et al. 2012). CLASH is a survey to image massive galaxy clusters in 16 bands using the ACS and WFC3 with FOVs of $4^{\prime} \times 4^{\prime}$ and $2^{\prime} \times 2^{\prime}$ on the HST. The CLASH images were released in two resolutions; 30 and 65 mas. We used the 65 mas resolution for the increased signal-to-noise ratio $(\mathrm{S} / \mathrm{N})$, especially in the WFC3, where the 30 mas images slightly oversample the point-spread function (PSF). Photometry was measured with SEXTRACTOR using the parameters FLUX_AUTO. Deblending parameters had to be carefully considered because initial photometry would be able to separate the clump from the galaxy, but deblending was necessary to remove the nearby foreground galaxy. This was also apparent from the publicly released CLASH catalogs of AS1063. We used the following SEXTRACTOR parameters: DEBLEND_ NTHRESH $=4$ and DEBLEND_MINCONT $=0.005$.

\subsection{Optical Spectroscopy}

Optical spectroscopy of AS1063 was obtained with the Low Dispersion Survey Spectrograph (LDSS-3) on the MagellanClay telescope on 2007 April 16 and 2007 July 15-16. LDSS-3 is an optical imager/spectrograph with a $4064 \times 4064$ STA0500A CCD. LDSS-3 was observed in multiobject mode using the VPH-all grating, covering a wavelength range of 3750-9500 $\AA$, with a resolution of $R=860$, an 8.3 diameter FOV, and a pixel scale of 0 ". 189 pixel $^{-1}$ along each slit. Spitzer/MIPS $24 \mu \mathrm{m}$ sources in AS1063 were selected for spectroscopy, including the three bright $24 \mu \mathrm{m}$ sources near the cluster center. Three masks were observed for the cluster using $1^{\prime \prime}$ width slits. For the mask containing AS1063a, the slit was aligned with the major axis of the galaxy based on the archival $H S T$ /WFPC2 imaging before the CLASH data set. Each mask was integrated for 45 minutes with conditions of 0 " 76,1 ."09, 0 ". 94 seeing (FWHM).

The LDSS-3 observations were reduced using the COSMOS data reduction package (Dressler et al. 2011). COSMOS performs flat-fielding, wavelength calibration, and sky subtraction, which produces a $2 \mathrm{D}$ spectrum for each object. The COSMOS data reduction package is based on an optical model in order to construct a wavelength solution, y-distortion, line curvature, and tilt for each slit. The sky-subtraction algorithm is based on the Kelson (2003) optimal sky subtraction, in which the sky subtraction is performed before rectifying the spectra. This method enables better removal of the sky lines and reduces the noise in the final spectra. Stacking of the final spectra is based on the positions of the alignment stars used in the mask. This ensures the maximal amount of flux per object, accounting for any possible movement in the instrument. The dome flats did not have adequate flux in the blue for correcting the slit-to-slit variation of the sensitivity function introduced by the VPH grating. It was necessary to use twilight sky flats to correct for the slit-to-slit variation. The 1D spectra were extracted with a 1.!9 aperture. For AS1063a, a 5!"7 aperture was used for the entire galaxy with smaller apertures for the individual regions of the galaxy. Then the line fluxes were measured by fitting a Gaussian to the emission lines and integrating the flux under the curve. The slit loss for the line flux is computed by convolving the HST ACS images at F606W and F814W by the LDSS-3 seeing and measuring the amount of flux lost going through the $1^{\prime \prime} \times 12^{\prime \prime}$ slit. Direct images of AS1063 were taken through the mask without the disperser, which were used to determine the position of the objects within the slits. 


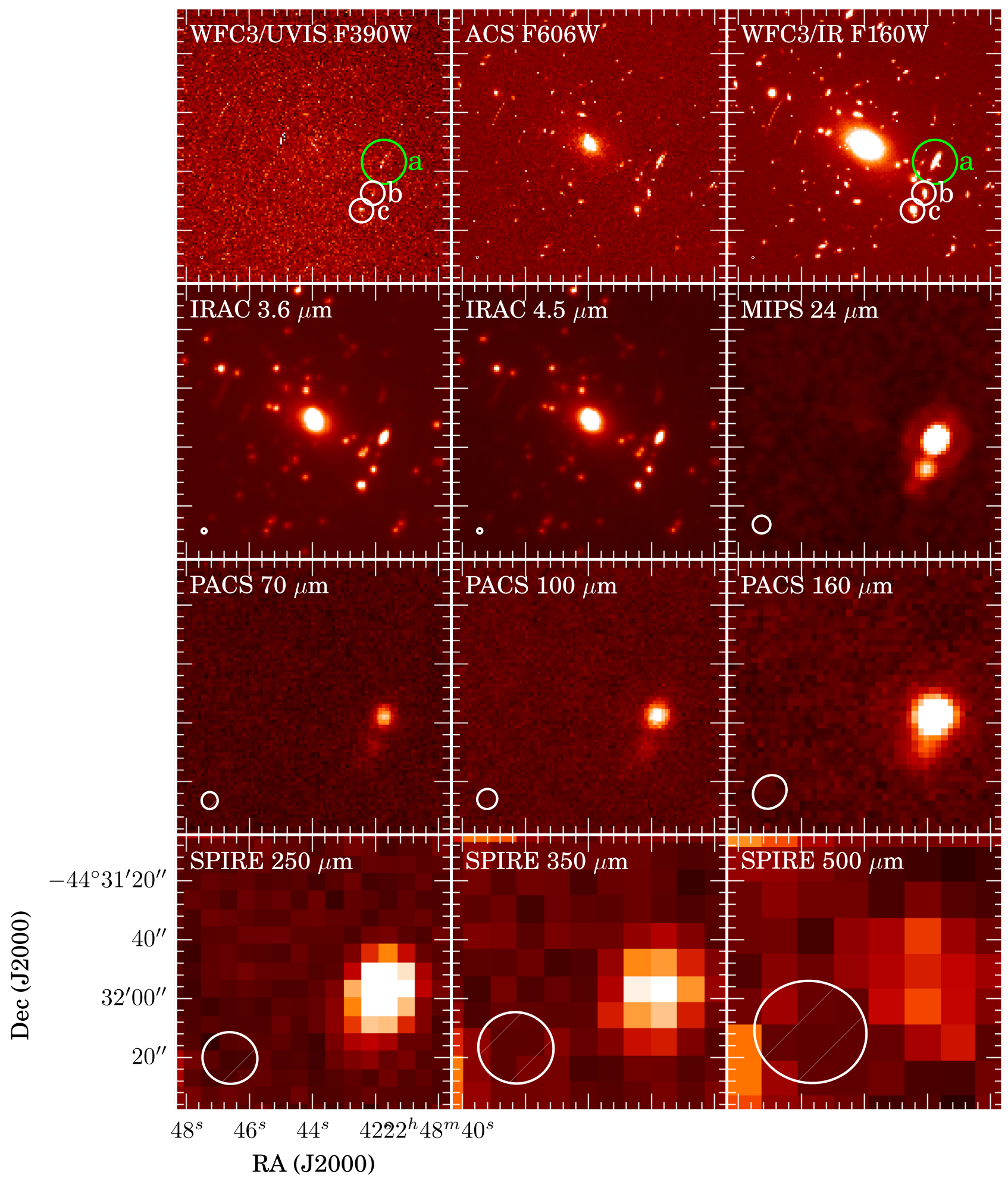

Figure 1. The cluster core of AS1063 imaged in HST (WFC3/UVIS F390W, ACS F606W, WFC3/IR F160W), Spitzer IRAC (3.6 and $4.5 \mu \mathrm{m})$ and MIPS $24 \mu \mathrm{m}$, Herschel PACS $(100$ and $160 \mu \mathrm{m})$, and SPIRE $(250,350$, and $500 \mu \mathrm{m})$. Each panel shows the central 1'.6 $\times 1$ 1'.6 area of the cluster core. The green circle marks the position of the lensed galaxy AS1063a. The lensed galaxy's far-IR/submillimeter spectral energy distribution is fully sampled by PACS and SPIRE. The white circles mark the positions of the two cluster galaxies $\left(z_{\text {spec }}=0.336\right)$, which can be seen in MIPS and PACS, but drop out of the SPIRE bands. The point-spread function of each of the instruments is marked in the bottom left corner in each panel.

\subsection{Near-IR Spectroscopy}

Near-IR spectroscopy of AS1063a was obtained with the MMT and Magellan Infrared Spectrograph (MMIRS; McLeod et al. 2012) on the Magellan-Clay telescope in long-slit mode on
2012 April 14, under poor seeing (>1"!66) and nonphotometric conditions. MMIRS is a near-IR imager/spectrograph with $2048 \times 2048 \mathrm{HgCdTe}$ Hawaii-2 detector. The spectra were taken with a 1 !' 2 width slit using the J grating with the $\mathrm{zJ}$ filter, 
covering a wavelength range of $0.94-1.51 \mu \mathrm{m}$, with a resolution of $R=800$, a $6.9 \times 6.9 \mathrm{FOV}$, and pixel scale of 0 !' 202 pixel $^{-1}$ along the slit. The spectra were taken in 5-minute exposures using a four-position dither pattern for four exposures, providing a total integration of 20 minutes on source. The long-slit alignment was with the major axis of AS1063a. The spectra were flat-fielded with dome flats and wavelength calibrated by $\mathrm{OH}$ skylines. Frames were subtracted from each other $(\mathrm{A}-\mathrm{B}$, $\mathrm{B}-\mathrm{A}$ ) in order to remove the sky emission from the source as well as the dark current. All the frames were median combined into one image using imcombine in IRAF (Tody 1986, 1993), with sigma clipping turned on. The 1D spectra were extracted with an $8^{\prime \prime}$ aperture. The line fluxes were measured by fitting a Gaussian to the emission lines and integrating the curve. Then they were corrected for slit loss by comparing the ratio of the HST images at F105W to the same image convolved by the MMIRS seeing. The slit loss for the line flux is computed by convolving the HST WFC3/IR images at F105W by the MMIRS seeing and measuring the amount of flux lost going through the slit.

HST WFC3/IR G102 and G141 grism spectra for AS1063 were obtained from the Grism Lens-Amplified Survey from Space (Schmidt et al. 2014; Treu et al. 2015). The grism data for AS1063 were reduced using AXE (Kümmel et al. 2009), which maps objects detected in the direct image to the objects in slitless grism spectra. The direct images were combined using MultiDrizzle. Sources in the direct image were detected using SExtractor, which is used as an input catalog for AXE. A contamination model is created from all the detected sources, including their zeroth-, first-, and second-order spectra. Tweakshifts is then used to determine their spatial offset between direct images for different visits but at the same roll angle. Then the grism spectra of multiple visits, at the same roll angle, were drizzled together. Finally, the AXE routines are run to drizzle the 2D spectra and extract the spectra with the options of slitless_geom and orient turned on. The 2D spectra are then divided by the instrument sensitivity function to flux calibrate the spectra. A sliding median with a window of 50 pixels $(1200 \AA$ ) was used to subtract off the continuum and contaminating sources from AS1063a. Apertures were placed over the emission lines in the 2D spectra to measure their fluxes.

\subsection{Far-IR/Submillimeter Imaging}

Herschel PACS (Poglitsch et al. 2010) images at 100 and $160 \mu \mathrm{m}$ were obtained for the core of AS1063 with an FOV of $9^{\prime} \times 9^{\prime}$ (Egami et al. 2010). The PACS instrument, operating in the dual-band photometry mode, consists of two bolometer arrays, a blue channel $(32 \times 64$ pixels $)$ and a red channel $(16 \times 32$ pixels $)$. The blue channel has two filters, $60-85 \mu \mathrm{m}$ and $85-125 \mu \mathrm{m}$, whereas the red channel has one, $125-210 \mu \mathrm{m}$, with each channel having an FOV of 1 .75 $\times 3$ '.5. Additional $70 \mu \mathrm{m}$ images were later obtained (PI: T. Rawle) on 2013 May 26, to better constrain the dust temperatures of warm cluster galaxies. Since PACS operates in a dual photometer mode, deeper $160 \mu \mathrm{m}$ images were also obtained with this program. The PACS maps were generated with UNIMAP (Piazzo et al. 2015) with a pixel scale of 1 "' 0,1 !" 0 , and 2 !" 0 .

Herschel SPIRE (Griffin et al. 2010) images at 250, 350, and $500 \mu \mathrm{m}$ were obtained for the field of AS1063 with an FOV of $23^{\prime} \times 26^{\prime}$. SPIRE in photometry mode has three bands, 250, 350 , and $500 \mu \mathrm{m}$, with the bands consisting of 139,88 , and 43 bolometers, respectively. Each band has an FOV of $4^{\prime} \times 8^{\prime}$.
The FWHM of the beam size at each band is $17 !$ " $6,23 !$ ! , and 35 !'2. SPIRE maps were generated using HIPE v10.0. The pixel scales of each of the maps are $6^{\prime \prime}, 9^{\prime \prime}$, and 12", respectively. SPIRE photometry was measured using IRAF task daophot.

Large APEX Bolometer Camera (LABOCA; Siringo et al. 2009) $870 \mu \mathrm{m}$ observations of AS1063 were part of the LABOCA Lensing Survey (E187A0437A,M-087.F-00052011). The LABOCA $870 \mu \mathrm{m}$ images of AS1063 have an FOV of $\sim 5$ ! 4 , with the center of the field $1^{\prime}$ north of the cluster center. The LABOCA beam is 24". 3 (FWHM). More details about the observation and reduction photometry can be found in Boone et al. (2013).

\section{Results}

\subsection{Three Bright $24 \mu \mathrm{m}$ Sources}

Figure 1 shows the Spitzer/IRAC (3.6 and $4.5 \mu \mathrm{m})$, Spitzer/ MIPS $(24 \mu \mathrm{m})$, Herschel/PACS $(70,100,160 \mu \mathrm{m})$, and Herschel/SPIRE $(250,350$, and $500 \mu \mathrm{m})$ images covering the central 1!.6 × 1!.6 area of the massive cluster AS1063. As the figure shows, the cluster core is surprisingly devoid of IR/ submillimeter sources, but three bright sources are clearly detected 24 " southwest of the brightest cluster galaxy. Their IRAC counterparts were unambiguously identified (marked with the green and white circles in Figure 1), and the brightest $24 \mu \mathrm{m}$ source in the north is seen to dominate the observed fluxes in the Herschel/PACS and SPIRE bands. The measured flux densities of these three bright $24 \mu \mathrm{m}$ sources are listed in Table 1.

Our follow-up optical spectroscopy showed that the brightest $24 \mu \mathrm{m}$ source (AS1063a) corresponds to a background galaxy at $z=0.61$, detecting Balmer emission $(\mathrm{H} \beta-\mathrm{H} 8)$, [O II] $\lambda 3727$, [O III] $\lambda \lambda 4959,5007$, and [Ne III]). The other two fainter $24 \mu \mathrm{m}$ sources (AS1063b and AS1063c) correspond to cluster galaxies at $z=0.347$, detecting $\mathrm{Ca}$ II $\mathrm{H}$ and $\mathrm{K}$ absorption and $\mathrm{H} \alpha$ and [N II] $\lambda \lambda 6548,6583$. The three $24 \mu \mathrm{m}$ sources are listed in Table 2, and their optical spectra are plotted in Figure 2. These redshifts are consistent with those published by Gómez et al. (2012).

The MMIRS near-IR spectrum of AS1063a detect $\mathrm{H} \alpha$ and [N II] $\lambda 6585$ lines (Figure 3) and the HST WFC3/IR G102 grism spectrum detects $\mathrm{H} \alpha$ blended with [N II] $\lambda \lambda 6548,6583$ lines (Figure 4). In addition, the grism also detects [S II] $\lambda 6717,6731$ and [S III] $\lambda 9069,9545$. The measured line fluxes are shown in Tables 3 and 4. The line fluxes corrected for slit loss are also given, as described in Sections 2.4 and 2.5. The $H S T$ WFC3/IR G102 grism observations of $\mathrm{H} \alpha+[\mathrm{N} \mathrm{II}]$ are consistent with the Magellan/MMIRS measurement of those lines.

AS1063a is detected in all three PACS bands (Figure 1). At 70 and $100 \mu \mathrm{m}$ it is distinctly identifiable, whereas at $160 \mu \mathrm{m}$ it becomes blended with AS1063b. The third bright source (AS1063c) is detected at $70 \mu \mathrm{m}$ and marginally detected at 100 and $160 \mu \mathrm{m}$. The sources were not severely crowded, and it was possible to get similar photometric values $(<7 \%$ difference) doing both aperture and PSF photometry. Aperture photometry was used at 70 and $100 \mu \mathrm{m}$, and PSF photometry was used at $160 \mu \mathrm{m}$.

At $250 \mu \mathrm{m} \mathrm{AS1063a} \mathrm{was} \mathrm{blended} \mathrm{with} \mathrm{the} \mathrm{nearby} \mathrm{cluster}$ galaxy AS1063b, and crowded field photometry (PSF fitting) was necessary to properly deblend the sources. The spectral 
Table 1

Photometry of Three Bright $24 \mu \mathrm{m}$ Sources

\begin{tabular}{|c|c|c|c|}
\hline & AS1063a & AS1063b & AS1063c \\
\hline Band & $\begin{array}{r}\text { Magnitude } \\
{[\mathrm{AB}]}\end{array}$ & $\begin{array}{r}\text { Magnitude } \\
{[\mathrm{AB}]}\end{array}$ & $\begin{array}{r}\text { Magnitude } \\
{[\mathrm{AB}]}\end{array}$ \\
\hline F225W & $21.48 \pm 0.05$ & $<22.88$ & $<23.41$ \\
\hline F275W & $21.01 \pm 0.03$ & $22.46 \pm 0.15$ & $22.24 \pm 0.08$ \\
\hline F336W & $20.96 \pm 0.05$ & $22.33 \pm 0.11$ & $21.90 \pm 0.05$ \\
\hline F390W & $20.94 \pm 0.07$ & $21.83 \pm 0.04$ & $21.67 \pm 0.04$ \\
\hline F435W & $20.68 \pm 0.06$ & $21.57 \pm 0.16$ & $21.37 \pm 0.06$ \\
\hline F475W & $20.55 \pm 0.08$ & $21.47 \pm 0.12$ & $20.85 \pm 0.07$ \\
\hline F606W & $19.81 \pm 0.04$ & $20.50 \pm 0.04$ & $19.86 \pm 0.03$ \\
\hline F625W & $19.58 \pm 0.06$ & $20.28 \pm 0.06$ & $19.62 \pm 0.04$ \\
\hline F775W & $19.10 \pm 0.04$ & $19.86 \pm 0.05$ & $19.22 \pm 0.03$ \\
\hline F814W & $19.01 \pm 0.05$ & $19.68 \pm 0.06$ & $19.13 \pm 0.04$ \\
\hline F850LP & $18.85 \pm 0.08$ & $19.52 \pm 0.09$ & $18.95 \pm 0.06$ \\
\hline F105W & $18.54 \pm 0.01$ & $19.30 \pm 0.02$ & $18.77 \pm 0.01$ \\
\hline F110W & $18.47 \pm 0.01$ & $19.16 \pm 0.02$ & $18.67 \pm 0.01$ \\
\hline F125W & $18.42 \pm 0.02$ & $19.02 \pm 0.03$ & $18.54 \pm 0.02$ \\
\hline F140W & $18.25 \pm 0.01$ & $18.89 \pm 0.01$ & $18.42 \pm 0.01$ \\
\hline F160W & $18.07 \pm 0.02$ & $18.75 \pm 0.02$ & $18.31 \pm 0.02$ \\
\hline Band & $\begin{array}{r}\text { Flux } \\
{[\mathrm{mJy}]}\end{array}$ & $\begin{array}{r}\text { Flux } \\
{[\mathrm{mJy}]}\end{array}$ & $\begin{array}{r}\text { Flux } \\
{[\mathrm{mJy}]}\end{array}$ \\
\hline $3.6 \mu \mathrm{m}$ & $0.35 \pm 0.03$ & $0.13 \pm 0.01$ & $0.18 \pm 0.02$ \\
\hline $4.5 \mu \mathrm{m}$ & $0.29 \pm 0.03$ & $0.12 \pm 0.01$ & $0.17 \pm 0.02$ \\
\hline $5.8 \mu \mathrm{m}$ & $0.37 \pm 0.04$ & $0.09 \pm 0.01$ & $0.10 \pm 0.01$ \\
\hline $8.0 \mu \mathrm{m}$ & $0.30 \pm 0.04$ & $0.22 \pm 0.02$ & $0.12 \pm 0.01$ \\
\hline $24 \mu \mathrm{m}$ & $2.22 \pm 0.02$ & $0.77 \pm 0.01$ & $0.27 \pm 0.03$ \\
\hline $70 \mu \mathrm{m}$ & $32.2 \pm 2.3$ & $9.0 \pm 0.5$ & $<3.1$ \\
\hline $100 \mu \mathrm{m}$ & $69.3 \pm 4.9$ & $14.5 \pm 1.0$ & $3.7 \pm 0.8$ \\
\hline $160 \mu \mathrm{m}$ & $105.8 \pm 7.5$ & $24.8 \pm 1.3$ & $<8.2$ \\
\hline $250 \mu \mathrm{m}$ & $69.3 \pm 6.8$ & $11.9 \pm 5.4$ & $<16.2$ \\
\hline $350 \mu \mathrm{m}$ & $36.1 \pm 6.2$ & $<17.1$ & $<17.1$ \\
\hline $500 \mu \mathrm{m}$ & $21.9 \pm 6.1$ & $<17.5$ & $<17.5$ \\
\hline
\end{tabular}

Note. Herschel errors listed are computed from the rms of the maps plus the calibration error, which is $5 \%$ in PACS and $4 \%$ in SPIRE. PACS and SPIRE flux limits were presented in Rawle et al. (2016).

Table 2

Spectroscopic Redshifts of Three Bright $24 \mu \mathrm{m}$ Sources

\begin{tabular}{lccccc}
\hline \hline Source & R.A. & Decl. & $z^{\mathrm{a}}$ & $z_{\text {quality }}^{\mathrm{b}}$ & ID $^{\mathrm{c}}$ \\
\hline AS1063a & $22: 48: 41.760$ & $-44: 31: 56.53$ & 0.611 & 4 & 30 \\
AS1063b & $22: 48: 42.113$ & $-44: 32: 07.39$ & 0.337 & 4 & 8 \\
AS1063c & $22: 48: 42.480$ & $-44: 32: 12.99$ & 0.337 & 4 & 9 \\
\hline
\end{tabular}

Notes.

a Spectroscopic redshift originally published in Gómez et al. (2012).

b $z_{\text {quality }}$ is described in the Appendix.

${ }^{\mathrm{c}}$ ID of galaxy used in Table 9 .

energy distribution (SED) for AS1063a is shown in Figure 5. Within the cluster field it was difficult to find bright wellisolated sources to measure the PSF of the image, so we used an empirical PSF provided by the Herschel Science Center. The empirical PSF was binned and rotated to the PA of Herschel when it observed AS1063 and after subtracting the sources from the map had a resulting rms of $\sim 5.9 \mathrm{mJy}$. At longer wavelengths ( 350 and $500 \mu \mathrm{m}$ ) the two cluster members are almost completely undetected.
In order to determine the optical/near-IR counterpart of a submillimeter source it is necessary to have several bands spanning a wide wavelength range between the optical and the submillimeter. Sources are traced from the submillimeter to the optical, stepping down in wavelength while ensuring that each source is being followed near the centroid of the original submillimeter source. Sometimes submillimeter sources may be blends of multiple IRAC and MIPS sources. If the sources are not too close (less than a pixel away), then they can be deblended using the IRAF task daophot with the IRAC and MIPS position priors. The redshift of the sources can also alleviate source confusion, where dust emission from lower redshift sources may fall below the detection limit for longer wavelength SPIRE bands.

A complete list of $24 \mu \mathrm{m}$ sources with spectroscopic redshifts within the deepest MIPS coverage of AS1063 $\left(5^{\prime} \times 5^{\prime}\right)$ is presented in the Appendix. A full analysis will be presented in a future paper.

\subsection{IR-luminous Lensed Galaxy at $\mathrm{z}=0.61$}

In the IR/submillimeter range, the most conspicuous source in the core of AS1063 is the IR-bright galaxy at $z=0.61$ (Figure 1). Its SED was fit using Chary \& Elbaz (2001) and Rieke et al. (2009) templates (Figure 5). Both template sets are based on SEDs of local galaxies. The best $\chi^{2}$ model produces a total IR luminosity, integrated from 8 to $1000 \mu \mathrm{m}$ (Kennicutt 1998), of $1.3 \times 10^{12} \mathrm{~L}_{\odot} \mu^{-1}$, where $\mu$ is the magnification factor. The dust temperature was determined to be $36 \pm 1 \mathrm{~K}$ by fitting a modified blackbody to the peak of the dust bump with $\beta$ fixed at 1.5, using Equation (1).

$$
S_{\nu}=N\left(\nu / \nu_{0}\right)^{\beta} B_{\nu}(T),
$$

where $B_{\nu}$ is a Planck function (evaluated at single temperature $T), N$ is the amplitude, $\nu$ is the frequency, $\nu_{0}$ is the frequency that is typically fixed at $c / 250 \mu \mathrm{m}$, and $\beta$ is the exponent that determines the shape of the modified blackbody.

The Spitzer/IRS spectrum of this galaxy is shown in Figure 6, and the measured line fluxes are listed in Table 5. The rest-frame mid-IR spectrum looks like that of a star-forming galaxy, with a strong polycyclic aromatic hydrocarbon (PAH) feature $(11.3 \mu \mathrm{m})$. The measured [Ne III] $(15.5 \mu \mathrm{m}) /[\mathrm{Ne}$ II] $(12.8 \mu \mathrm{m})$ line ratio is $<1(0.33 \pm 0.06)$, a value typical of a solar-metallicity starburst galaxy (Thornley et al. 2000; Rigby $\&$ Rieke 2004). It is therefore clear that the predominant source of the IR luminosity is star formation and not an active galatic nucleus (AGN). This is consistent with the fact that the measured optical line ratios put this galaxy on the edge of the star-forming sequence/composite region in the Baldwin, Phillips, \& Terlevich (BPT; Baldwin et al. 1981) diagram (Figure 7).

The HST images show that this galaxy has a spiral-like morphology with clearly defined bulge and disk components (Figure 8). Our optical spectrum obtained along the long axis of the galaxy shows a velocity offset from one side to the other (Figure 9), caused by the rotation of the disk component. The proximity of the $z=0.61$ galaxy to the cluster center suggests that its gravitational magnification is likely significant, although its normal-looking morphology of a spiral galaxy indicates that the magnification effect is not large enough to destroy the intrinsic galaxy morphology, essentially stretching the galaxy in the direction tangential to the cluster center. 

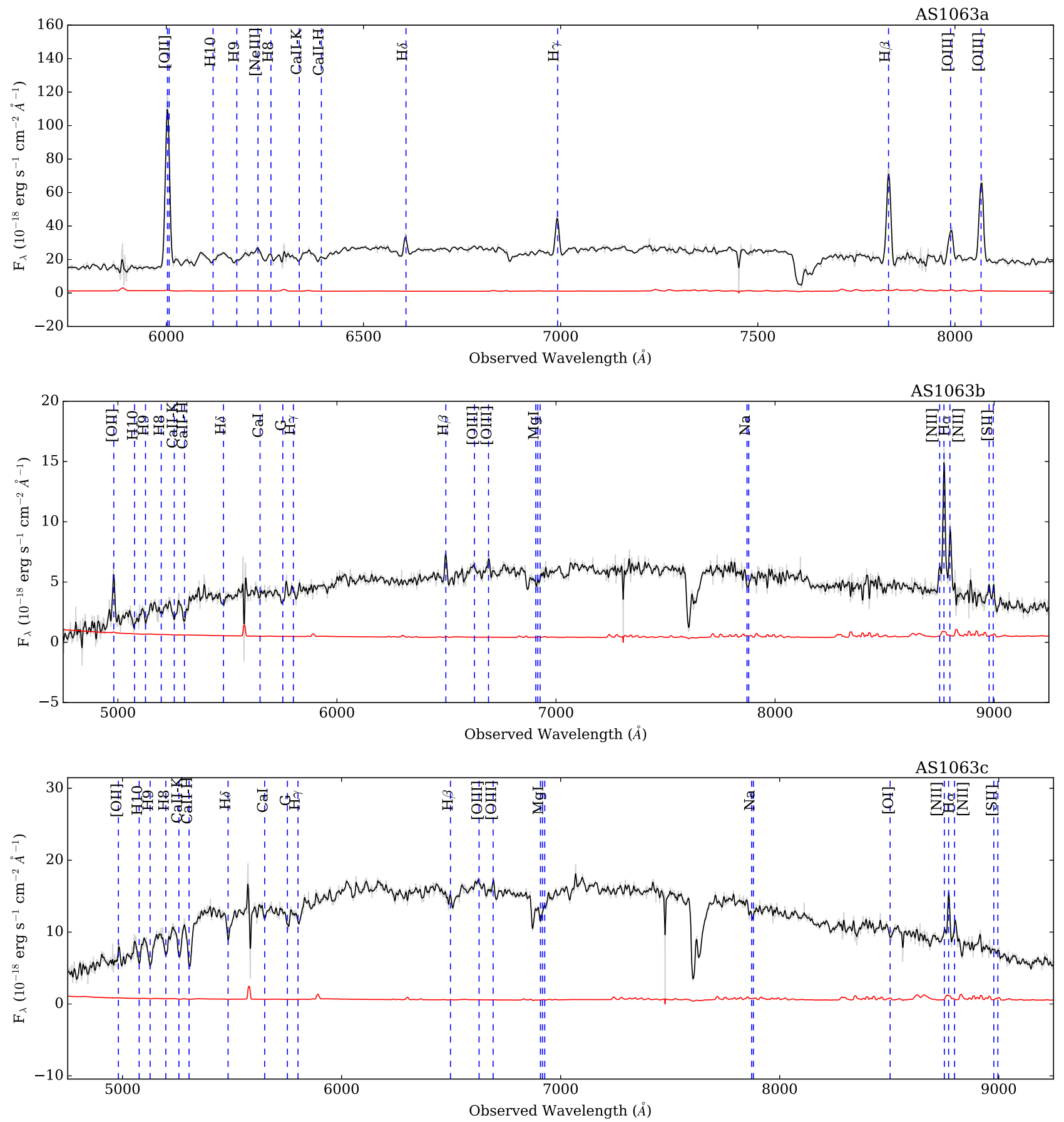

Figure 2. Magellan/LDSS-3 optical spectra of the three bright $24 \mu \mathrm{m}$ sources in AS1063. Top: multiple Balmer lines (H $\alpha-\mathrm{H} 8)$ are detected in AS1063a suggesting it has undergone a recent burst of star formation. Middle and bottom: we detect $\mathrm{H} \alpha$ in the cluster members (AS1063b and AS1063c) suggesting that they may be falling into the cluster for the first time.

Figure 1 clearly shows that the brightest IR/submillimeter emission comes from this lensed galaxy at $z=0.61$. It is, however, not clear which part of the galaxy is exactly responsible for this strong IR emission. Figure 8 shows that the peak of the brightest $24 \mu \mathrm{m}$ emission is located 1!"27 southsoutheast from the galaxy nucleus, falling in the middle of the disk. No bright optical/near-IR counterpart is seen at the peak of the $24 \mu \mathrm{m}$ emission, although three smaller star-forming clumps are seen nearby in the $H S T$ images. The $24 \mu \mathrm{m}$ emission appears resolved in one axis, elongated along the longer axis of the galaxy, suggesting the possibility that the
$24 \mu \mathrm{m}$ emission may originate from multiple components in the galaxy.

\subsubsection{Bright Optical Clump with Strong Line Emission}

What is most striking about the $z=0.61$ lensed galaxy, which corresponds to the brightest MIPS $24 \mu \mathrm{m}$ and PACS/ SPIRE source, is its exceptionally bright optical clump seen at one edge of the disk, 2" 48 southeast from the center of the galaxy. The CLASH HST data show that this clump is the brightest feature in all of the HST/ACS optical bands. In the 

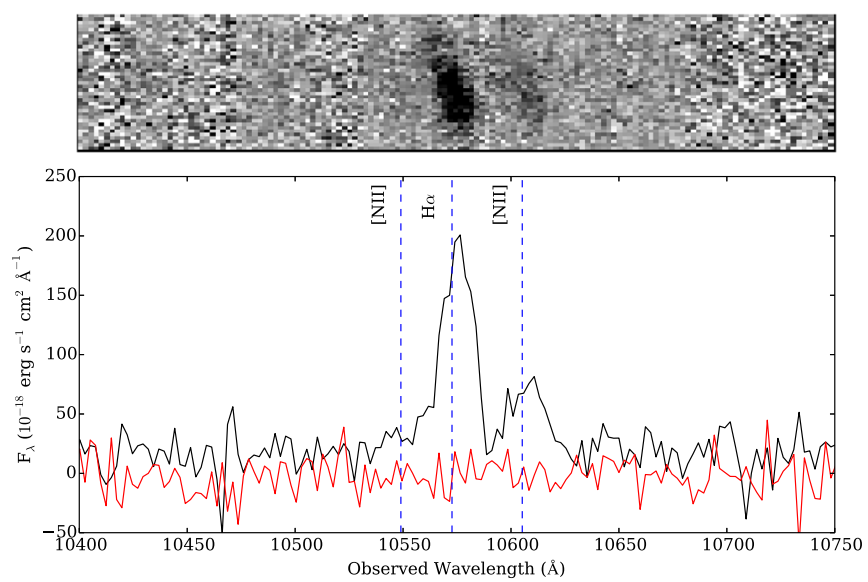

Figure 3. Magellan/MMIRS spectum of AS1063a, H $\alpha$, and $[\mathrm{N}$ II] $\lambda 6585$ are clearly detected within 20 minutes of integration. The asymmetry of the line is the result of the velocity offset of the bright clump at the edge of the galaxy. Even though the magnification is increasing across the galaxy toward the clump (southeast direction), it is not a major contributor to the asymmetry.

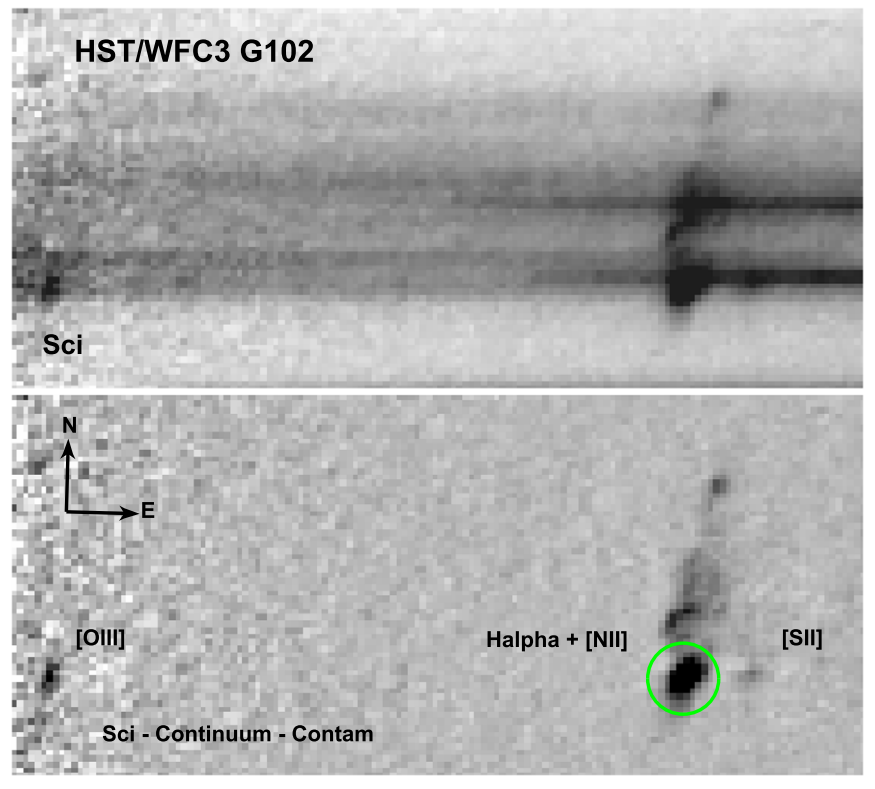

Figure 4. HST WFC3/IR G102 spectrum of the lensed galaxy AS1063a. Top: the reduced AXE spectrum. Bottom: the reduced $\mathrm{AXE}$ spectrum with AS1063a's continuum removed, along with the contaminating sources by using a sliding median. The green circle marks the position of the star-forming region. $\mathrm{H} \alpha+[\mathrm{N} \mathrm{II}] \lambda \lambda 6548,6583$ are detected throughout the entire galaxy, while [O III] $\lambda 5007$ and [S II] $\lambda \lambda 6717,6731$ are only detected for the starforming region.

HST/WFC3 near-IR bands, however, the central bulge becomes the dominant feature.

This bright optical clump appears to have quite a significant size intrinsically. By fitting an elliptical Gaussian to the HST WFC3/IR F105W image (which corresponds to rest-frame $\mathrm{H} \alpha$ ) and subtracting in quadrature a Gaussian PSF measured in the same image, we have derived the spatial size of this clump as $0.547 \pm 0$ ". $025 \times 0.099 \pm 0$ ". 034 . This corresponds to $3686 \pm 171 \mathrm{pc} \times 670 \pm 230 \mathrm{pc}$ at $z=0.61$ if we do not take into account the lensing effect.

The 2D spectra of the $z=0.61$ galaxy shown in Figures 4 and 9 show that the bright clump emits strongly in line emission (e.g., Balmer lines $\mathrm{H} \alpha-\mathrm{H} 8$, forbidden lines [O II] $\lambda 3727,[\mathrm{O} \mathrm{III}] \lambda \lambda 4959,5007, \quad[\mathrm{Ne} \mathrm{III}], \quad[\mathrm{S} \mathrm{III}]$ and [S III]).
Table 3

Optical and Near-IR Emission Line Fluxes of AS1063a

\begin{tabular}{|c|c|c|}
\hline Line & $\begin{array}{c}\text { Flux } \\
\left(10^{-17} \mathrm{erg} \mathrm{s}^{-1} \mathrm{~cm}^{-2}\right)\end{array}$ & $\begin{array}{c}\text { Flux }_{\text {corr }}^{\mathrm{a}} \\
\left(10^{-17} \mathrm{erg} \mathrm{s}^{-1} \mathrm{~cm}^{-2}\right)\end{array}$ \\
\hline \multicolumn{3}{|c|}{ Entire Galaxy } \\
\hline$[\mathrm{O}$ II $] \lambda 3727$ & $123.8 \pm 1.5$ & $132.1 \pm 1.6$ \\
\hline $\mathrm{H} \delta$ & $10.3 \pm 0.8$ & $11.0 \pm 0.9$ \\
\hline $\mathrm{H} \gamma$ & $25.9 \pm 1.1$ & $27.7 \pm 1.2$ \\
\hline $\mathrm{H} \beta$ & $74.4 \pm 2.0$ & $80.0 \pm 2.1$ \\
\hline$[\mathrm{O} \mathrm{III]}] \lambda 4959$ & $33.0 \pm 2.8$ & $35.3 \pm 3.1$ \\
\hline$[\mathrm{O}$ III $] \lambda 5007$ & $67.4 \pm 1.7$ & $72.0 \pm 1.8$ \\
\hline $\mathrm{H} \alpha$ & $308.1 \pm 8.2$ & $348.8 \pm 9.3$ \\
\hline$[\mathrm{N} \mathrm{II}] \lambda 6583$ & $137.7 \pm 6.9$ & $155.9 \pm 7.8$ \\
\hline \multicolumn{3}{|c|}{ H II Region } \\
\hline$[\mathrm{O}$ II] $] 3727$ & $61.6 \pm 0.8$ & $65.8 \pm 0.9$ \\
\hline$[\mathrm{Ne}$ III] $] \lambda 3869$ & $2.9 \pm 0.6$ & $3.1 \pm 0.6$ \\
\hline $\mathrm{H} 8$ & $4.2 \pm 0.5$ & $4.5 \pm 0.5$ \\
\hline $\mathrm{H} \epsilon$ & $2.8 \pm 0.4$ & $3.0 \pm 0.4$ \\
\hline $\mathrm{H} \delta$ & $7.0 \pm 0.4$ & $7.5 \pm 0.4$ \\
\hline $\mathrm{H} \gamma$ & $17.1 \pm 0.5$ & $18.4 \pm 0.5$ \\
\hline $\mathrm{H} \beta$ & $42.3 \pm 0.7$ & $45.5 \pm 0.8$ \\
\hline$[\mathrm{O} \mathrm{III]}] \lambda 4959$ & $15.5 \pm 0.7$ & $16.7 \pm 0.8$ \\
\hline$[\mathrm{O}$ III $] \lambda 5007$ & $48.3 \pm 0.6$ & $51.9 \pm 0.6$ \\
\hline \multicolumn{3}{|c|}{ Bulge } \\
\hline$[\mathrm{O}$ II $] \lambda 3727$ & $40.2 \pm 0.9$ & $43.0 \pm 1.0$ \\
\hline $\mathrm{H} \delta$ & $2.9 \pm 0.4$ & $3.1 \pm 0.4$ \\
\hline $\mathrm{H} \gamma$ & $8.4 \pm 0.7$ & $9.0 \pm 0.7$ \\
\hline $\mathrm{H} \beta$ & $25.5 \pm 1.1$ & $27.3 \pm 1.2$ \\
\hline$[\mathrm{O}$ III $] \lambda 4959$ & $7.4 \pm 1.4$ & $7.9 \pm 1.5$ \\
\hline$[\mathrm{O}$ III $] \lambda 5007$ & $14.1 \pm 0.8$ & $15.1 \pm 0.9$ \\
\hline \multicolumn{3}{|c|}{ Spiral Arm } \\
\hline [O II] $] \lambda 3727$ & $9.1 \pm 0.5$ & $9.7 \pm 0.5$ \\
\hline $\mathrm{H} \gamma$ & $1.3 \pm 0.3$ & $1.4 \pm 0.3$ \\
\hline $\mathrm{H} \beta$ & $2.4 \pm 0.6$ & $2.6 \pm 0.6$ \\
\hline [O III] $] \lambda 5007$ & $2.4 \pm 0.3$ & $2.6 \pm 0.3$ \\
\hline
\end{tabular}

Note.

${ }^{\text {a }}$ Flux corrected for slit loss by convolving the HST ACS and WFC/IR images by the LDSS-3 and MMIRS seeing and measuring the amount of light that is blocked by the slit.

Table 4

$H S T /$ WFC3 G102 and G141 Spectra of AS1063a

\begin{tabular}{|c|c|}
\hline Line & $\begin{array}{c}\text { Flux } \\
\left(10^{-17} \mathrm{erg} \mathrm{s}^{-1} \mathrm{~cm}^{-2}\right)\end{array}$ \\
\hline \multicolumn{2}{|c|}{ Entire Galaxy } \\
\hline $\mathrm{H} \alpha+[\mathrm{N}$ II $] \lambda \lambda 6548,6583$ & $545.5 \pm 2.4$ \\
\hline \multicolumn{2}{|c|}{ H II Region } \\
\hline$[\mathrm{O}$ III $] \lambda 5007$ & $53.2 \pm 4.1$ \\
\hline $\mathrm{H} \alpha+[\mathrm{N} \mathrm{II}] \lambda \lambda 6548,6583$ & $275.0 \pm 1.2$ \\
\hline$[\mathrm{S}$ II] $\lambda \lambda 6717,6731$ & $15.1 \pm 0.6$ \\
\hline [S III] $\lambda 9069$ & $4.4 \pm 0.6$ \\
\hline$[\mathrm{S}$ III $] \lambda 9545$ & $32.5 \pm 0.7$ \\
\hline \multicolumn{2}{|c|}{ Bulge } \\
\hline $\mathrm{H} \alpha+[\mathrm{N} \mathrm{II}] \lambda \lambda 6548,6583$ & $205.1 \pm 1.5$ \\
\hline \multicolumn{2}{|c|}{ Spiral Arm } \\
\hline $\mathrm{H} \alpha+[\mathrm{N} \mathrm{II}] \lambda \lambda 6548,6583$ & $50.5 \pm 1.2$ \\
\hline
\end{tabular}




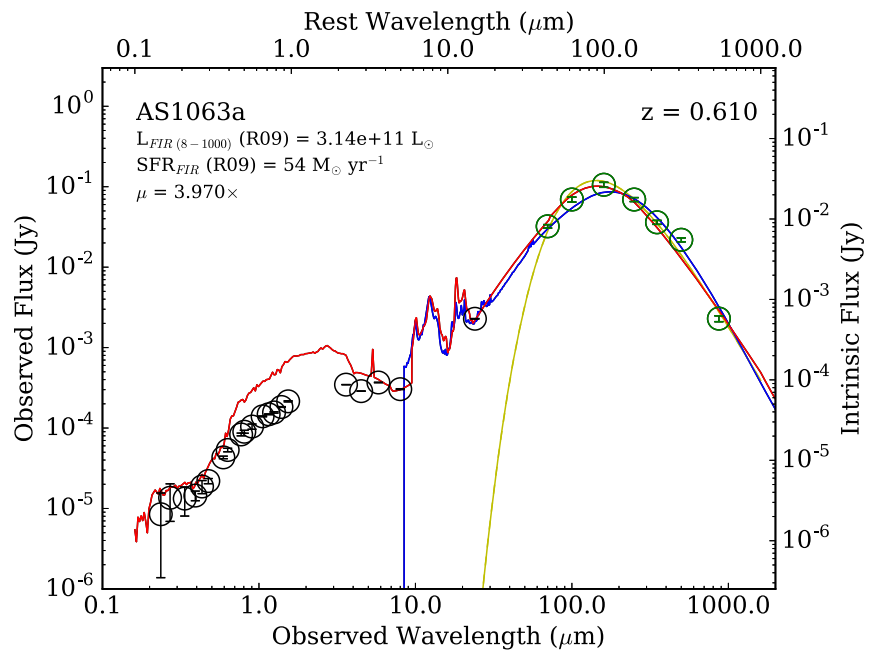

Figure 5. SED of the lensed galaxy AS1063a in the core of AS1063. Fits were made to the Herschel PACS 70, 100, $160 \mu \mathrm{m}$ and Herschel SPIRE 250, 350, 500 and LABOCA $870 \mu \mathrm{m}$ (green circles). The photometry blueward of the $70 \mu \mathrm{m}$ was ignored in the fitting of the SED (black circles). The lines show the best-fitting templates to the far-IR/submillimeter data. The blue line is the Rieke et al. (2009) templates, and the red line is the Chary \& Elbaz (2001) templates. The yellow line shows the best-fitting modified blackbody. On the right axis, the observed flux was corrected based on the magnification $(4.0 \pm 0.1)$ determined by the lens modeling by LENSTOOL (Jullo et al. 2007).

Although we do detect the line emission throughout the galaxy disk, it quickly becomes fainter away from the clump, while the $\mathrm{Ca}$ II $\mathrm{H}$ and $\mathrm{K}$ absorption features become more prominent, reflecting an increasing light contribution from an older stellar population in the bulge and spiral arm area on the other side of the galaxy (Figure 9).

\section{Discussion}

\subsection{Lens Model Reconstruction}

In order to determine the intrinsic properties of AS1063a it was necessary to remove the effects of gravitational lensing. Modeling of the gravitational lens, in the strong regime, was done with LENSTOOL (Kneib et al. 1996; Jullo et al. 2007; Jullo \& Kneib 2009). LENSTOOL models the cluster lenses by utilizing a nonparametric method, using multiple images and redshifts of lensed background galaxies in order to constrain the model. In the newest CLASH data, we identified five multiply imaged systems, one of which was confirmed with a spectroscopic redshift, that were used as input constraints for the lens model (Richard et al. 2014; B. Clément et al. 2019, in preparation). With the lens model it was possible to determine critical lines and spatial and flux magnifications of the lensed galaxy.

From the lens model we are able to reconstruct AS1063a in the source plane (Figure 10, right panel). Most of the magnification is linear, with very little distortion.

The luminosity-weighted magnification is $4.0 \pm 0.1$. The error in the magnification is the statistical error and does not include systematics such as choice in parameterization/ modeling or use of bad constraints/assumptions in the model. AS1063a is $\sim 5^{\prime \prime}$ from the critical line, which suggests that is too far for differential magnification to be a significant factor.

There is a slight gradient in the spatial distortion of the galaxy, from the southern edge to the northern edge, $3.6 \times-$ $2.6 \times$ in linear magnification. In ACS this resolves objects

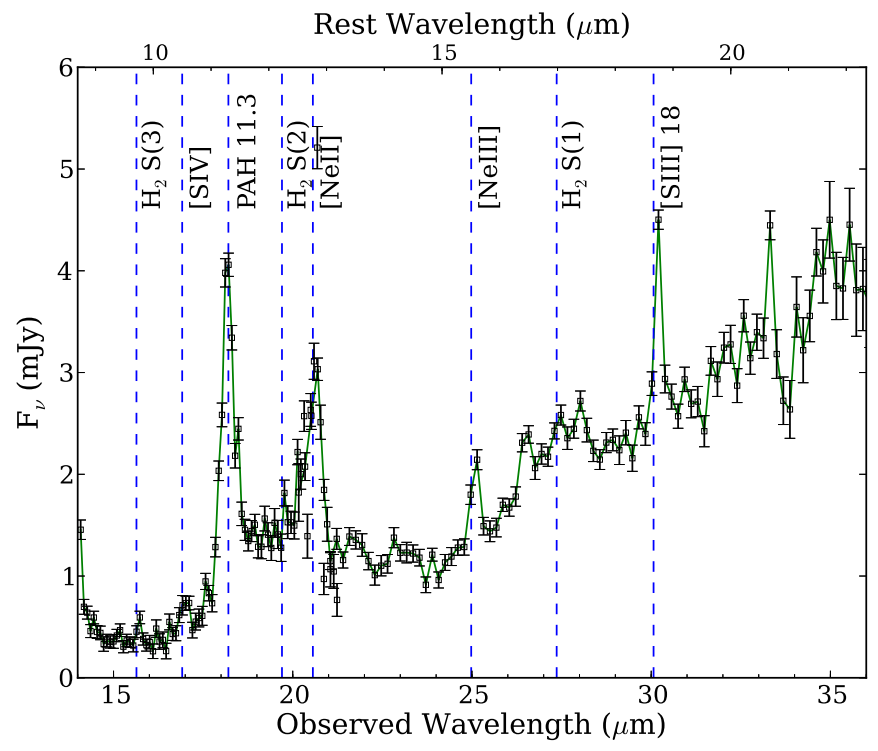

Figure 6. The Spitzer/IRS spectrum of the lensed galaxy AS1063a. We observe the [Ne III] and [Ne II] lines, which we use to determine whether our galaxy contains an AGN.

Table 5

Mid-IR Line Fluxes of AS1063a

\begin{tabular}{lcc}
\hline \hline Line & $\begin{array}{c}\text { Wavelength } \\
(\mu \mathrm{m})\end{array}$ & $\begin{array}{c}\text { Flux } \\
\left(10^{-17} \mathrm{erg} \mathrm{s}^{-1} \mathrm{~cm}^{-2}\right)\end{array}$ \\
\hline$[\mathrm{Ar} \mathrm{III}]$ & 9.0 & $7 \pm 15$ \\
$\mathrm{H}_{2} \mathrm{~S}(3)$ & 9.7 & $47 \pm 11$ \\
{$[\mathrm{~S} \mathrm{IV}]$} & 10.5 & $62 \pm 13$ \\
$\mathrm{H}_{2} \mathrm{~S}(2)$ & 12.2 & $38 \pm 15$ \\
{$[\mathrm{Ne} \mathrm{II]}$} & 12.8 & $238 \pm 16$ \\
{$[\mathrm{Ne} \mathrm{III]}$} & 15.5 & $78 \pm 13$ \\
$\mathrm{H}_{2} \mathrm{~S}(1)$ & 17.0 & $32 \pm 13$ \\
{$[\mathrm{~S} \mathrm{IIII} 18$} & 18.7 & $167 \pm 8$ \\
\hline
\end{tabular}

larger than 90-130 pc, and WFC3/IR 240-340 pc. The LDSS3 spectrum, at 0 !'76 seeing, spatially resolves $1.4-2.0 \mathrm{kpc}$. The flux amplification from the southern edge to the northern edge is $4.5 \times-3.1 \times$, as shown in the right panel in Figure 10 . The bright star-forming clump corresponds to the region of the larger amplification at the southern edge of the galaxy.

The bulge of AS1063a is prominent in the F775W filter and longward wavelengths. When comparing the observed image to the reconstructed image, the galaxy only appears stretched in the observed image (Figure 10, left). From the reconstructed image a few noticeable features pop out; there are two spiral arms, a bulge, and multiple bright clumps (star-forming knots) with one very prominent bright clump (the giant $\mathrm{H}$ II region). The magnification at the giant bright clump is $4.44 \times$.

We discovered that there is also disagreement with the lens model constructed by Gómez et al. (2012). A redshift was obtained for lens B and lens C as designated by Gómez et al. (2012), which is one of the main constraints for the nonparametric lens model. According to our lens model, based on the location of the bright arc, AS1063a or lens A, it is well outside the $z=0.61$ critical line and no additional images are expected for this arc. The suggested counter image to lens A, counterpart $\mathrm{A}$, is another lensed arc. With the additional resolution provided by the CLASH data, it is clear that the colors do not match the colors of the bright arc, and the bright clump seen in the 


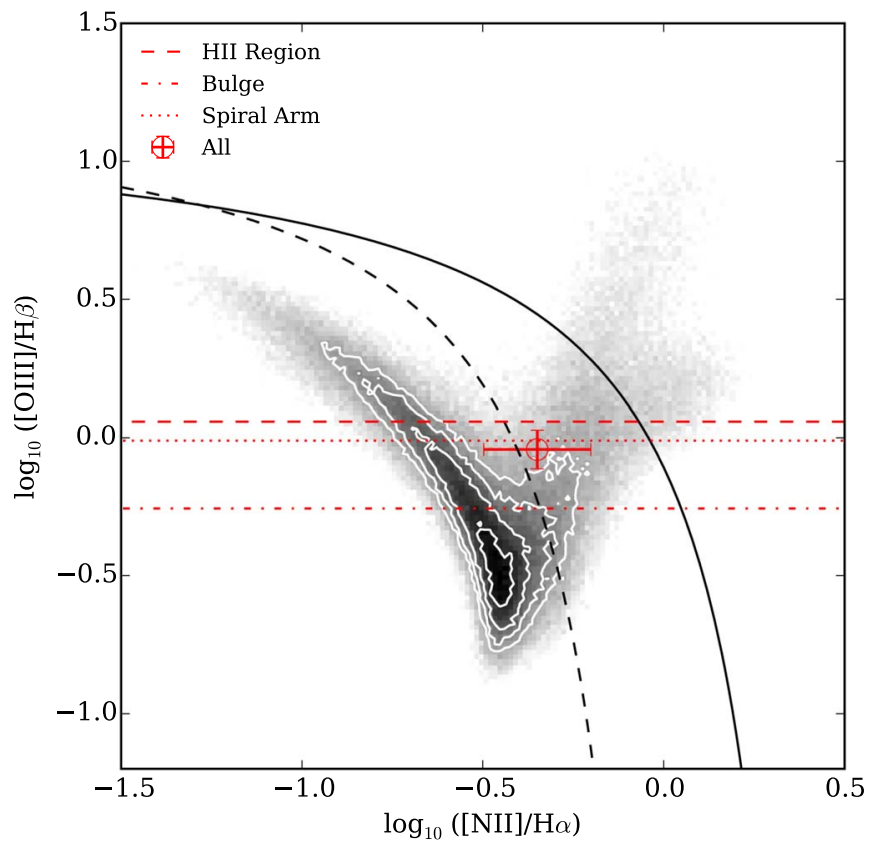

Figure 7. The BPT diagram of the three regions within the lensed galaxy AS1063a. The grayscale points are a 2D histogram of SDSS measured line ratios of redshifts in $z=0.005-0.25$. The red lines mark the [O III] $/ \mathrm{H} \beta$ ratios of three regions of the galaxy constrained by the optical data. Follow-up nearIR observations in poor seeing conditions roughly constrain the $[\mathrm{N} \mathrm{II}] / \mathrm{H} \alpha$ ratio of the entire galaxy. The black lines mark the Kewley et al. (2001) and Kauffmann et al. (2003) predictions for active galaxy type. The error bars include a $20 \%$ systematic error in the flux calibration.

counterpart A is not the bright clump identified in the bright arc, but is highly suggestive of a cluster galaxy.

\subsection{Physical Properties of the $\mathrm{z}=0.61$ Lensed IR-luminous Galaxy}

\subsubsection{SFRs}

When corrected for a magnification factor of $4.0 \pm 0.1$, the intrinsic IR luminosity of the $z=0.61$ galaxy becomes $(3.1 \pm$ $0.1) \times 10^{11} \mathrm{~L}_{\odot}$, with a corresponding SFR of $54 \pm 2 \mathrm{M}_{\odot} \mathrm{yr}^{-1}$. This means that this galaxy is intrinsically IR luminous and of the LIRG type (luminous IR galaxy, $L_{\mathrm{IR}} \geqslant 10^{11} \mathrm{~L}_{\odot}$ ).

On the other hand, the SFR derived from $\mathrm{H} \alpha$, detected with MMIRS, is significantly smaller. The observed $\mathrm{H} \alpha$ line luminosity gives an SFR of $28 \pm 1 \mathrm{M}_{\odot} \mathrm{yr}^{-1}$. Corrected for magnification, the value decreases to $7.0 \pm 0.3 \mathrm{M}_{\odot} \mathrm{yr}^{-1}$. When corrected for a visual extinction of $A_{\mathrm{V}}=1.5 \pm 0.2 \mathrm{mag}$ $(E(B-V)=0.36 \pm 0.04)$ derived from the Balmer decrement assuming case B recombination and using a Calzetti dust law, the value increases to $33 \pm 1 \mathrm{M}_{\odot} \mathrm{yr}^{-1}$, but this is still smaller than the IR-derived value of $54 \pm 2 \mathrm{M}_{\odot} \mathrm{yr}^{-1}$. This implies that $40 \%$ of the star formation is obscured.

The HST grism spectrum of AS1063a is at a spatial resolution of 0 "' 13 . At this spatial resolution we can determine the contributions for the spatially distinct components of the galaxy (i.e., H II region, bulge, and spiral arm). In order to utilize this information, we need to assume an $[\mathrm{N} \mathrm{II}] / \mathrm{H} \alpha$ ratio. From the MMIRS spectrum for the entire galaxy, we measure a ratio of $0.45 \pm 0.03$, which we assume for each of the regions in the galaxy. Unfortunately, the seeing and $\mathrm{S} / \mathrm{N}$ of the MMIRS spectrum are not sufficient enough to determine the ratio for the spatially distinct components of the galaxy. We also assume that the ratio [N II] $\lambda 6583 /[\mathrm{N} \mathrm{II}]$ $\lambda 6548 \sim 3$. With this we estimate the $\mathrm{H} \alpha$ flux for the entire galaxy as $(340.9 \pm 26.3) \times 10^{-17} \mathrm{erg} \mathrm{s}^{-1} \mathrm{~cm}^{-2} ; 171.9 \pm$ $13.3 \mathrm{erg} \mathrm{s}^{-1} \mathrm{~cm}^{-2}$ for the $\mathrm{H}$ II region, $128.2 \pm 9.9 \mathrm{erg} \mathrm{s}^{-1} \mathrm{~cm}^{-2}$ for the bulge, and $31.6 \pm 2.5 \mathrm{erg} \mathrm{s}^{-1} \mathrm{~cm}^{-2}$ for the spiral arm. Using the $E(B-V)$ computed for the entire galaxy and correcting for the magnification of each of the regions (i.e., $4.4 \times, 3.9 \times$, and $3.3 \times$ ), we find that the SFRs for each of the regions are the following: $14 \pm 1 \mathrm{M}_{\odot} \mathrm{yr}^{-1}$ for the $\mathrm{H}$ II region, $12 \pm 1 \mathrm{M}_{\odot} \mathrm{yr}^{-1}$ for the bulge, and $4 \pm 1 \mathrm{M}_{\odot} \mathrm{yr}^{-1}$ for the spiral arm.

In Section 4.2.3, $E(B-V)$ is also computed from the SED fitting of the photometry for AS1063a and its H II region, which are 0.31 and 0.18 , respectively. If we assume these $E(B-V)$ values and magnification corrections, it would affect the HST grism-based SFRs in the following way: $27 \pm 2 \mathrm{M}_{\odot} \mathrm{yr}^{-1}$ for AS1063a and $8 \pm 1 \mathrm{M}_{\odot} \mathrm{yr}^{-1}$ for the $\mathrm{H}$ II region. The overall $E(B-V)$ computed for the galaxy from the Balmer decrement agrees with the one computed from the photometry. However, for the H II region, it might be expected that there could be some geometry or sight-line effect that could result in a different $E(B-V)$ value. In particular, it is lower in this case, which is evident by the $\mathrm{H}$ II region being the brightest feature in the galaxy in the UV-optical bands as well as detecting more Balmer transitions (i.e., $\mathrm{H} \alpha-\mathrm{H} 8$ ). For the $\mathrm{H}$ II region we adopt the $\mathrm{SFR}=8 \pm 1 \mathrm{M}_{\odot} \mathrm{yr}^{-1}$ that is derived using the $E(B-V)$ value from the photometry, which seems more consistent with the evidence.

Using the $\mathrm{H} \beta$ and $\mathrm{H} \gamma$ and $\mathrm{H} \delta$ lines seen in the 2D optical spectrum (Figure 9), we can derive visual extinctions and extinction-corrected SFRs for spatially distinct components in the galaxy. However, the $E(B-V)$ values disagree with the one derived from $\mathrm{H} \alpha$ and $\mathrm{H} \beta$, increasing with higher order Balmer transitions. Upon further inspection it appears that self absorption from the continuum could be affecting the flux measured for the higher order Balmer lines.

\subsubsection{Precise Location of the IR Source}

As we mentioned in Section 3.2 the MIPS $24 \mu \mathrm{m}$ emission is offset from the bulge of the $z=0.61$ galaxy by 1 .'27 and elongated, suggesting it is resolved in one axis. One concern is whether the offset is real or whether it is a result of astrometric error due to the large MIPS $24 \mu \mathrm{m}$ PSF. The native pixel scale of the MIPS $24 \mu \mathrm{m}$ is 2 !" 45 pixel $^{-1}$. However, the image we use is sampled at 1.245 pixel $^{-1}$. In order to compare the bulge position to the MIPS position we need a set of unresolved sources that can be used for astrometry. For the $z=0.61$ lensed galaxy, the bulge can be identified in the F814W filter, which is also convenient due to the larger FOV of ACS. We used two methods to identify stars in the HST F814W images: (1) plotting the difference in the magnitude of two different sized apertures and (2) plotting magnitude versus half-light radius at a fixed aperture. Stars will resemble the PSF of the instrument for a wide range of radii, whereas if we are just using an FWHM measure, some galaxies may have FWHM similar to a star and lead to a false-positive detection. As an additional check, we visually inspected stars to ensure sources were not misidentified and were not blended with other sources. For sources we believe to be stars, we checked to see whether they are detected in IRAC and also to ensure they are not blended. From our clean list of point sources, we compute the separation angle between the sources detected in ACS and IRAC. We then measure the standard deviation of all the separations, computing the $1 \sigma$ error in the astrometric position of $0 . " 12$. 


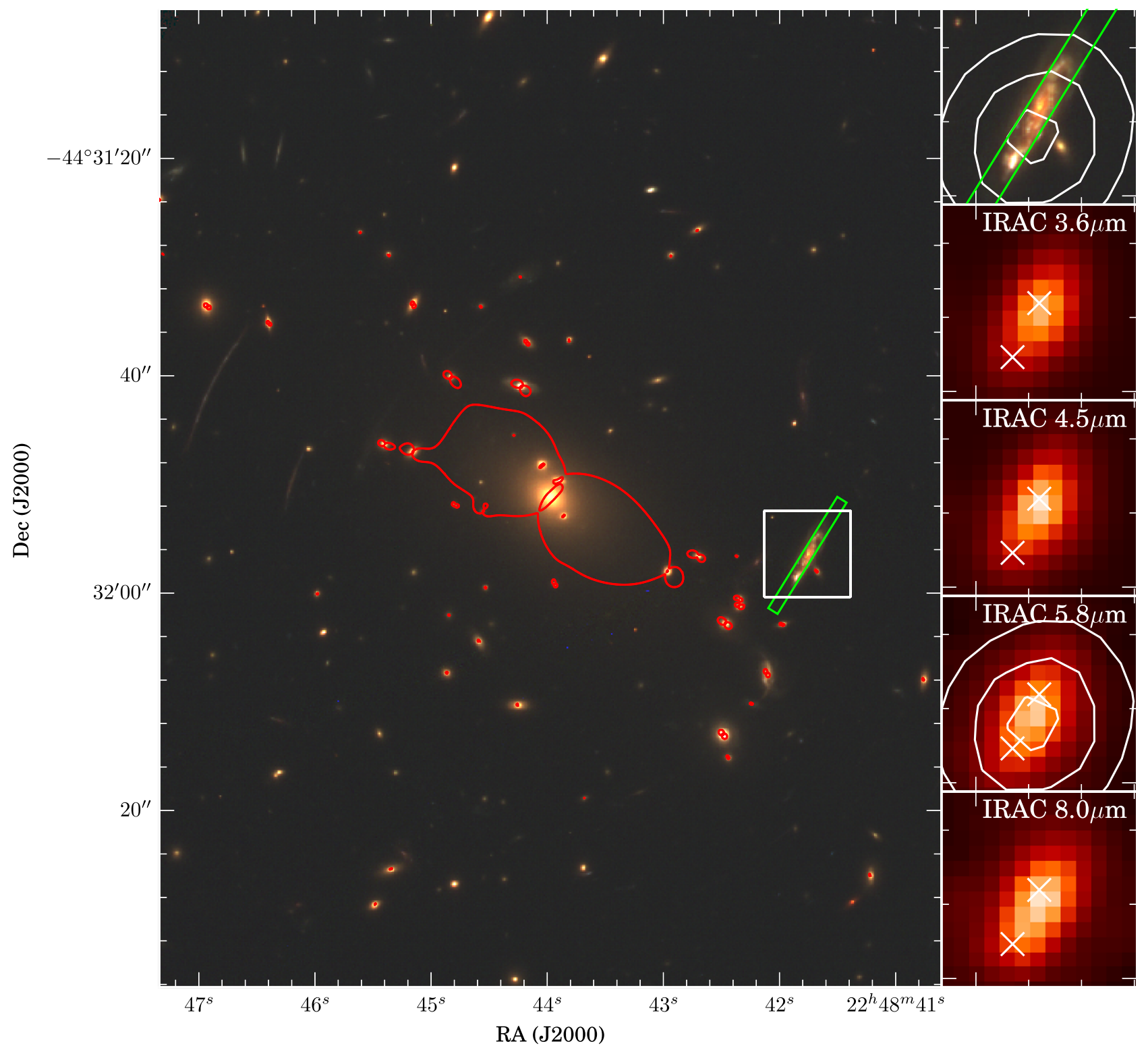

Figure 8. Left: three-color image of the cluster core AS1063 using HST ACS F606W, and HST WFC3/IR F110W and F160W filters. The main panel shows the central $1.0 \times 1 ! 2$ area of the cluster core. The red line marks the critical line at $z=0.61$. The green box marks the position of the slit used for optical spectroscopy of lensed galaxy AS1063a. The white box in the left panel shows the FOV of the source AS1063a displayed in the right panels. Right: the top right panel shows the zoomed HST three-color image, using the same filters at the left panel. The bottom four panels show each of the Spitzer/IRAC 3.6, 4.5, 5.8, and 8.0 $\mu$ m images with the same FOV as the top right panel. The white contour marks the position of the MIPS $24 \mu$ m contour. White x's mark the positions of the bright star-forming region and bulge.

At $24 \mu \mathrm{m}$ many of the sources are unresolved, very few of which are actually stars. In order to determine the astrometric error between IRAC and MIPS we rely on compact sources that are unresolved in IRAC. We use a similar strategy to the one described above to select point sources in IRAC. We compute the standard deviation of the separation angle between the positions in IRAC to the positions in MIPS and find an error of 0 !"23. The combined $1 \sigma$ error in the astrometric positions in ACS, IRAC, and MIPS is 0 "! 26 . At $4.9 \sigma$ we can confidently say that the offset of the MIPS peak emission is real.

In order to explain the elongation of the source in MIPS $24 \mu \mathrm{m}$, we simulate the source by convolving a Gaussian with the MIPS PSF. We then assume the position is at the center of the peak MIPS emission and vary the amplitude while minimizing $\chi^{2}$. We find that a single source at the peak of the $24 \mu \mathrm{m}$ emission is not sufficient to account for all the flux in the source, which has significant residual wings. To account for all the flux it appears that more than one Gaussian is needed. We next assume that the far-IR emission could be coming from two locations, offset from the peak emission. Two reasonable locations are the bulge of the galaxy and the starforming region. We find that fitting two Gaussians at those locations works well. The residuals are much smaller, with the majority of the flux being accounted for. The solution that works the best is two Gaussians with roughly the same amplitude. However, these solutions are highly degenerate and additional Gaussians could potentially be added for better fits. We can confidently say that it is unlikely that a single source is responsible for all of the far-IR emission at the position of the $24 \mu \mathrm{m}$ emission peak. There must be more than one source 


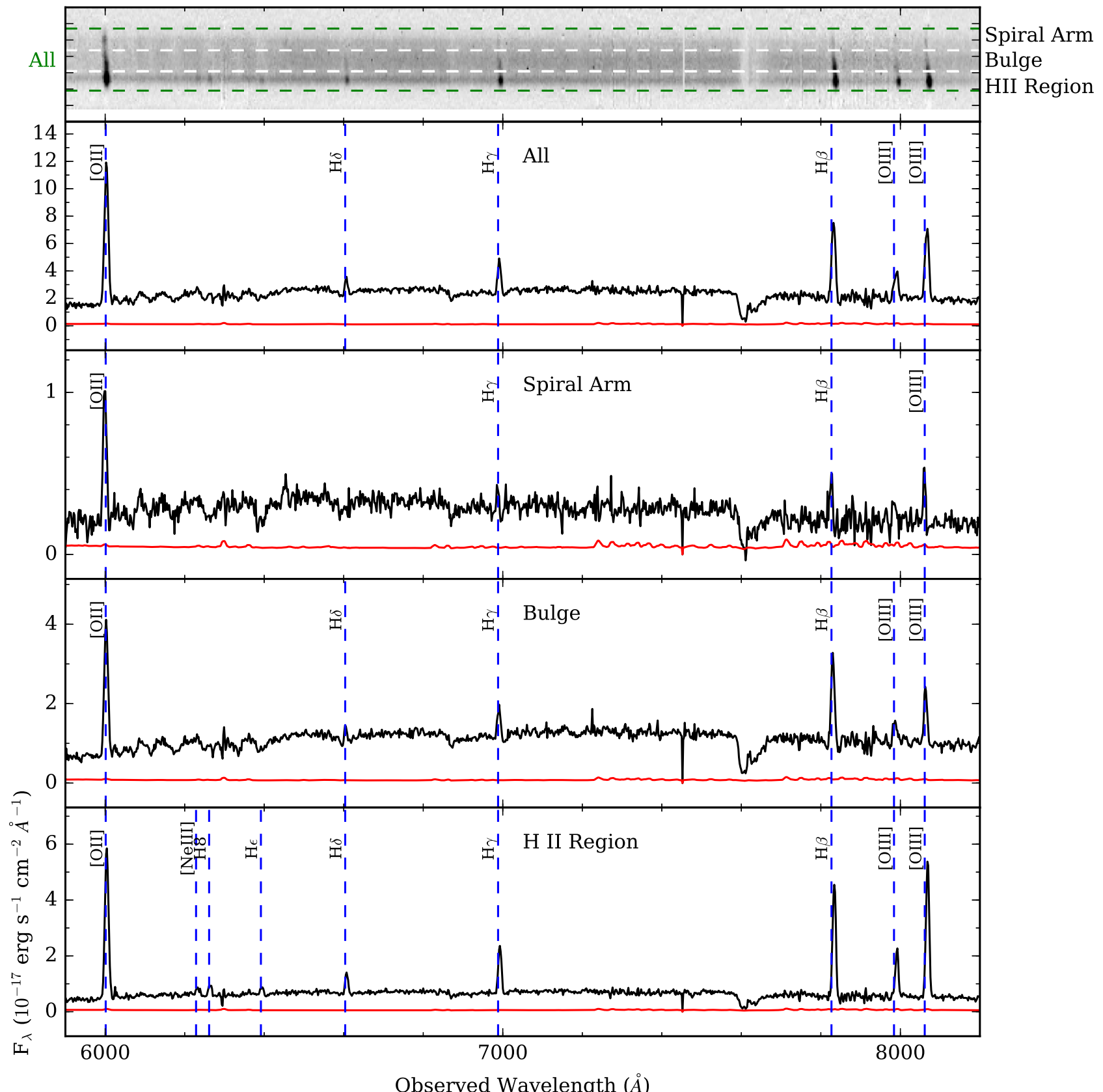

Figure 9. First panel: the 2D spectrum of the lensed galaxy AS1063a taken with Magellan's LDSS-3. Second panel: the 1D spectrum of the entire galaxy. Third panel: the 1D spectrum of the spiral arm. There appears to be an older stellar population; the Balmer lines are the weakest in this part of the galaxy. Fourth panel: the 1D spectrum of the bulge. This region corresponds to the central region of the $2 \mathrm{D}$ spectrum and the central region of the lensed galaxy. This region appears to have a significant older stellar population. Fifth panel: the 1D spectrum of the $\mathrm{H}$ II region. This region corresponds to the lower bright part of the 2D spectrum. This is the bright star-forming clump that is dominated by strong Balmer and forbidden emission lines.

contributing to the far-IR flux; higher spatial resolution is needed to resolve the situation.

Looking at the IRAC bands, there is a slight bump at $5.8 \mu \mathrm{m}$. Based on the redshift of this galaxy, it is expected that the $3.3 \mu \mathrm{m}$ PAH feature would be completely in the band. However, the $3.3 \mu \mathrm{m}$ PAH feature is much weaker than the other PAH features found in star-forming galaxies, and the bump we see in the SED may not be caused by this feature. It is expected that the majority of light contributing to the flux in the IRAC bands is from stars. If there is flux coming from the $3.3 \mu \mathrm{m}$ PAH feature, we would need to subtract off the stellar light. To account for the stellar light, we scale the flux in IRAC 3.6 to the IRAC $5.8 \mu \mathrm{m}$ image and subtract them from each other. We also do the same with the IRAC $4.5 \mu \mathrm{m}$ image. In each case there appears to be no evidence for residual flux coming from the $3.3 \mu \mathrm{m}$ PAH feature. If the bump seen in the SED is real, it is possible that the PAH emission could be throughout the galaxy at a low enough level that it might not be seen in the residuals. It appears unlikely that the flux would be originating from an individual region in the galaxy; otherwise it should have been seen in the residuals.

\subsubsection{Stellar Mass}

We fit the HST 16-band photometry to Bruzual \& Charlot (2003) models following Pérez-González et al. $(2008,2013)$ in 

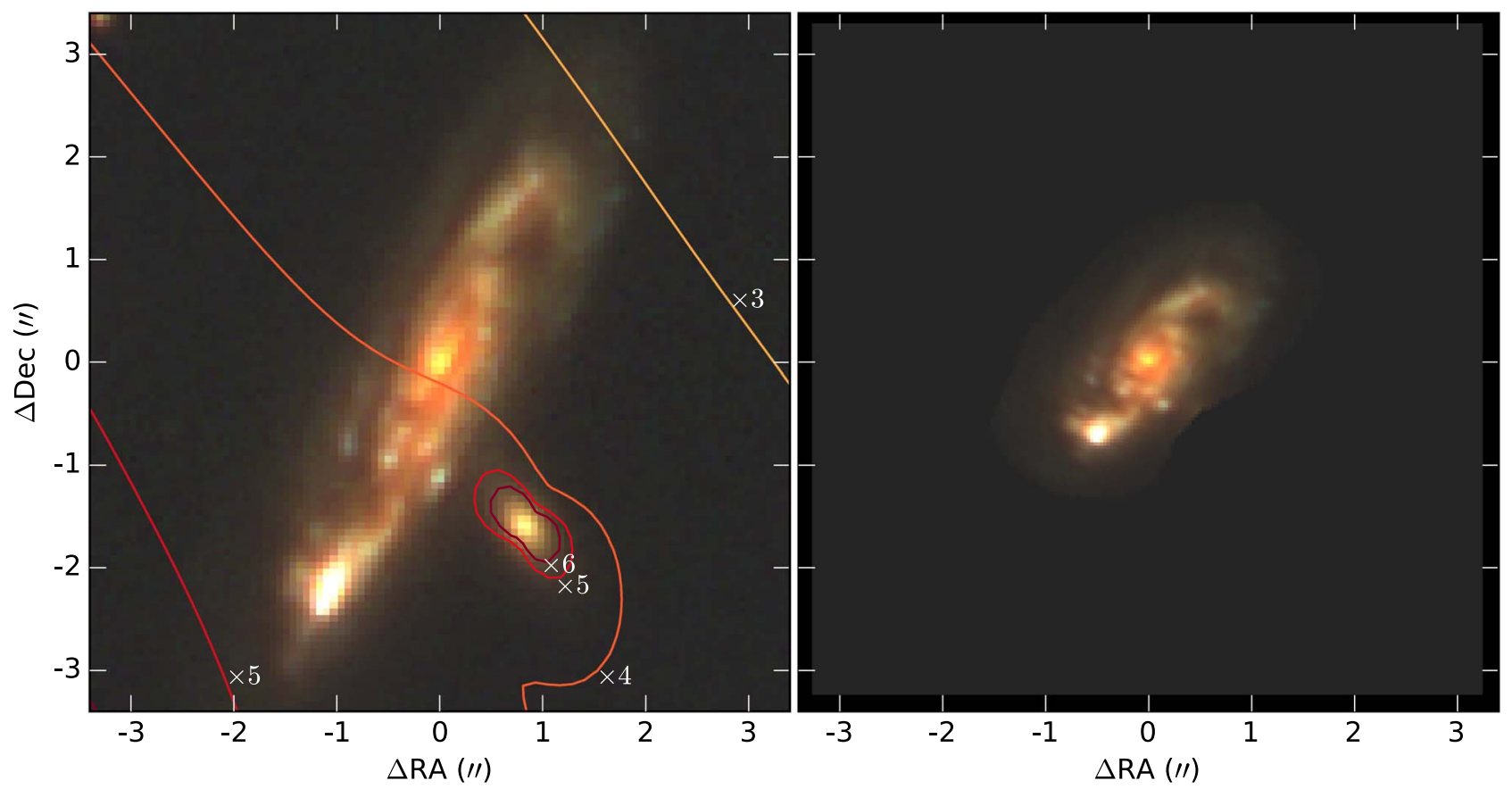

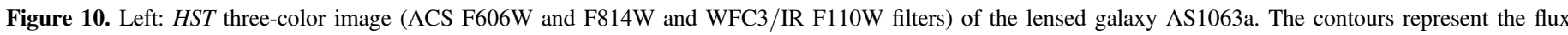

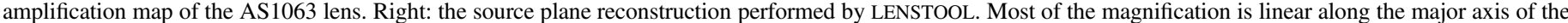

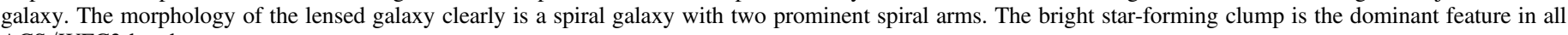
ACS/WFC3 bands.

order to determine stellar mass, dust attenuation, and stellar age and population. We assume a Chabrier initial mass function (IMF), Calzetti extinction, and exponentially decreasing star formation history. The models were run fixing the metallicity to solar metallicity and also allowing it to be a free parameter. The SED fitting was conducted on the photometry of AS1063a and just the $\mathrm{H}$ II region. The images used for the photometry of the $\mathrm{H}$ II region were PSF matched to ensure that we are comparing the same physical region in each band. We used TINYTIM (Krist et al. 2011) to generate the PSF for each of the 16 bands, convolved with the charge diffusion PSF and rebinned to the CLASH pixel scale of 65 mas. The IRAF task ps fmatch was used to convolve all of the $H S T$ images to the WFC3/F160W PSF.

The results of the SED fitting can be found in Table 6 and Figure 11. The stellar masses derived from the SED fitting are good to within 0.2 dex, including uncertainties in the IMF and other systematics. AS1063a's stellar mass appears to be unaffected by metallicity as its mass varies by $1.1 \times$ for the metallicities considered. In Section 4.2.5 we describe the metallicity derived from the nebular emission lines in which we find the value to be about solar metallicity. For AS1063a we adopt the stellar mass $\left(2.6 \times 10^{10} \mathrm{M}_{\odot}\right)$ at solar metallicity. The $\mathrm{H}$ II region on the other hand seems to vary by a factor of $1.5 \times$ depending on the metallicity. We also adopt the solar metallicity value $\left(1.1 \times 10^{9} \mathrm{M}_{\odot}\right)$, in which it appears to be close to solar metallicity, as we go over in Section 4.2.5.

When comparing the HII region in AS1063a to ones at higher redshift we find that it is about average stellar mass and that the high redshift $\mathrm{H}$ II regions span $10^{7}-10^{10} \mathrm{M}_{\odot}$. Looking at the samples individually, the H II regions in RCSGA0327 (Wuyts et al. 2014), a galaxy at $z=1.7$, fall on the lower mass side from $10^{7}$ to $10^{8} \mathrm{M}_{\odot}$, whereas the Wisnioski et al. (2012) and Förster Schreiber et al. (2011b) samples are on the higher mass range $10^{9}-10^{10} \mathrm{M}_{\odot}$ and $10^{8}-10^{10} \mathrm{M}_{\odot}$. This can be mostly
Table 6

Stellar Mass of AS1063a

\begin{tabular}{lccc}
\hline \hline $\begin{array}{l}\text { Metallicity } \\
{\left[\mathrm{Z}_{\odot}\right]}\end{array}$ & $A_{v}$ & $\begin{array}{c}\text { Age } \\
{[\mathrm{Gyr}]}\end{array}$ & $\begin{array}{c}\text { Stellar Mass } \\
{\left[\mathrm{M}_{\odot}\right]}\end{array}$ \\
\hline \multicolumn{4}{c}{ Entire Galaxy } \\
\hline 1.000 & $1.25_{-0.48}^{+0.21}$ & $0.65_{-0.27}^{+0.48}$ & $2.6_{-1.0}^{+1.5} \times 10^{10}$ \\
0.005 & $1.61_{-0.19}^{+0.18}$ & $0.64_{-0.21}^{+0.53}$ & $3.0_{-1.1}^{+1.7} \times 10^{10}$ \\
\hline \multicolumn{3}{c}{ H II Region } \\
\hline 1.000 & $0.74_{-0.24}^{+0.20}$ & $0.38_{-0.17}^{+0.31}$ & $10.5_{-3.9}^{+6.1} \times 10^{8}$ \\
0.200 & $1.15_{-0.41}^{+0.30}$ & $0.10_{-0.07}^{+0.16}$ & $7.1_{-2.6}^{+4.1} \times 10^{8}$ \\
\hline
\end{tabular}

explained by the Wisnioski et al. (2012) and Förster Schreiber et al. (2011b) samples being unlensed, so only the largest, most massive $\mathrm{H}$ II regions are probed, whereas the Wuyts et al. (2014) sample is a single lensed galaxy probing small H II regions at high spatial resolution.

Using the stellar mass at solar metallicity for AS1063a and the SFR derived from the far-IR, we compute the specific SFR (sSFR), which is $2.11 \mathrm{Gyr}^{-1}$. We use the analytic fitting function from Whitaker et al. (2012) to determine the sSFR of the star-forming main sequence, which is $0.35 \mathrm{Gyr}^{-1}$ for a galaxy with the same stellar mass and redshift as AS1063a. Galaxies with sSFRs $4 \times$ the star-forming main sequence are considered starbursts (Noeske et al. 2007; Rodighiero et al. 2011). The starburst region for a galaxy similar to AS1063a is $1.38 \mathrm{Gyr}^{-1}$, which means that AS1063a is a starburst.

\subsubsection{Kinematics}

Spectroscopically, this bright clump appears like an H II region embedded in a rotating disk, with a rotational velocity $V_{\max }$ of $134 \pm 17 \mathrm{~km} \mathrm{~s}^{-1}$ at $R=5.9 \mathrm{kpc}$, which is also found 

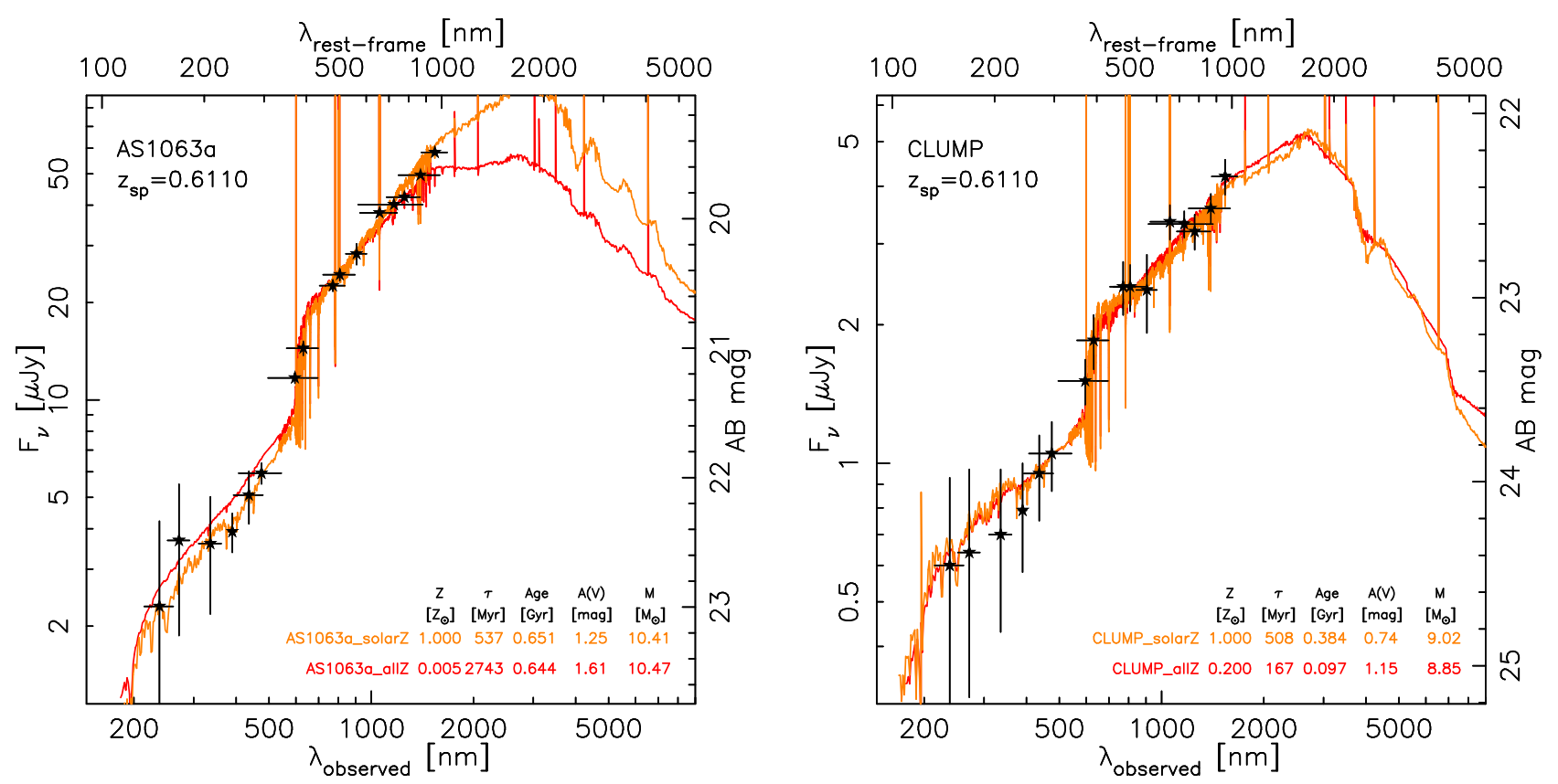

Figure 11. Left: SED fits to the intrinsic flux of the entire galaxy, AS1063a. Right: SED fits to the intrinsic flux of H II region in AS1063a. The templates used assume a Chabrier IMF, Calzetti extinction, and exponentially decreasing star formation history. The orange line shows solar metallicity template, and the red line is the bestfitting metallicity. From the photometry fitting, for both AS1063a and the clump, neither model is preferred. The metallicity determined from the nebular emission lines from the R23 and N2 diagnostics helps constrain which model is the appropriate one, that is, the solar metallicity model.

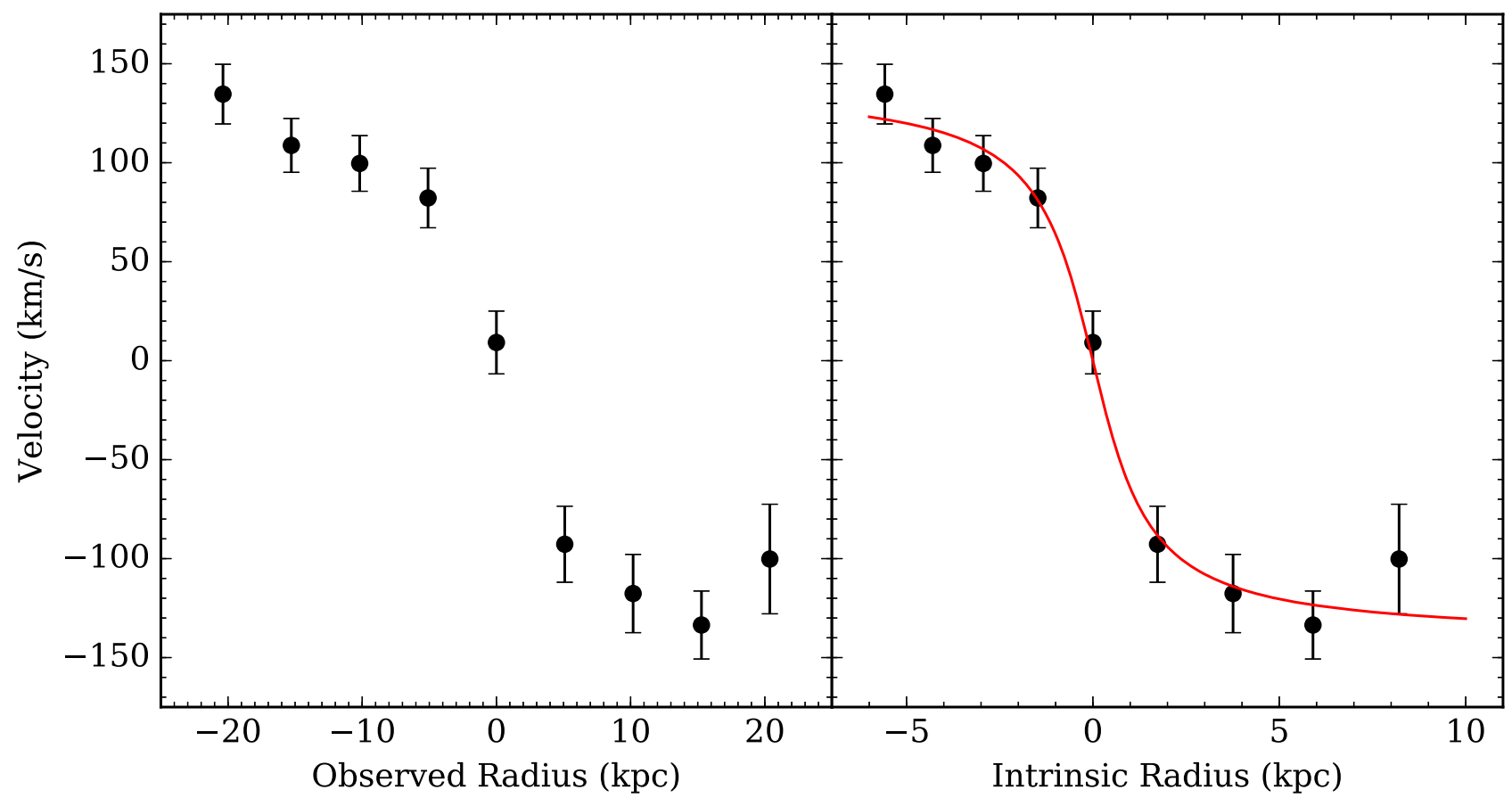

Figure 12. Left: the kinematics of the observed [O II] emission line measured from the 2D spectrum. We measured the centroid of the blended [O II] $\lambda \lambda 3726,3729$ doublet, which corresponds to $[\mathrm{O} \mathrm{II}] \lambda 3727$. [O II] is the brightest feature spatially with the highest $\mathrm{S} / \mathrm{N}$. In order to reduce the noise in the measurement, we binned the data into bins of 4 pixels each $\left(0{ }^{\prime \prime} 76\right)$, which is comparable to the seeing. Right: the intrinsic kinematics of the lensed galaxy AS1063a. The red line is the best-fit velocity profile. The galaxy appears to have a slight inclination, which is seen by a velocity gradient, suggesting that the galaxy is rotating as one system. The rotation curve appears to be flattening out outside $2 \mathrm{kpc}$.

by Gómez et al. (2012) and Karman et al. (2015). The clump itself shows strong Balmer emission $(\mathrm{H} \alpha-\mathrm{H} 8)$ and forbidden line emission ([O II $] \lambda 3727$, [O III] $] \lambda \lambda 4959,5007$, [Ne III]), and its systemic velocity falls on the rotation curve. AS1063a has a smooth rotation curve that flattens out outside of $2 \mathrm{kpc}$ (Figure 12).
A system in the local universe with a similar appearance to AS1063a is NGC 5194 (M51), which is a spiral galaxy interacting with another galaxy (NGC 5195). Sofue et al. (1999) looked at the rotation curves of local galaxies and found that NGC 5194 had a peculiar rotation curve (non-Keplerian). NGC3034 (M82), a starburst galaxy interacting with M81, also 
shows a peculiar rotation curve, in which it has a steep Keplerian decline. In the local universe it is expected that an isolated spiral galaxy should have a flat rotation curve at large radii $(5-30 \mathrm{kpc})$. Significant bumps or deviations from Keplerian rotation in the rotation curve could indicate an interaction with another galaxy or underlying substructure (subhalos). Kinematically it is difficult to completely rule out AS1063a as a merger; however, it appears unlikely.

From the rotation curve we can also measure a dynamical mass. In order to compute an accurate dynamical mass we need to determine the inclination of the galaxy. Using the source plane reconstruction of the galaxy, we measure the axis ratio (b/a) using GALFIT (Peng et al. 2002, 2010). We find an axis ratio of $b / a=0.502 \pm 0.007$. Assuming the galaxy is an oblate spheroid (Holmberg 1958), we use Equation (2) to compute the inclination:

$$
\cos ^{2} i=\frac{(b / a)^{2}-q^{2}}{1-q^{2}},
$$

where $i$ is the inclination angle, $b / a$ is the axis ratio ( $a$ is the semimajor axis, $b$ is the semiminor axis of the galaxy), and $q$ is the axis ratio for an edge-on galaxy. Typically $q$ is 0.13 in spiral galaxies. In order to understand the uncertainty in the inclination angle we performed a Markov chain Monte Carlo using 1000 realizations of the AS1063 lens model (LENSTOOL routine bayescleanlens), with each realization reconstructing AS1063a and the HST PSF in the source plane. We then use GALFIT to fit each of the realizations of the reconstructed source plane image, using the reconstructed PSF associated with a reconstructed image. The standard deviation of the PA of the galaxy was 1:5. Even with this deviation in the PA, we find that the uncertainty in the inclination is 0.5 . When we vary the parameter $q$, the disk thickness, from 0.11 to 0.2 (Courteau 1997), which is typical for spiral galaxies, we find that it varies by 1.5 . Given the systematic uncertainties for determining inclinations of galaxies (e.g., Barnes \& Sellwood 2003), we adopt $\pm 5^{\circ}$ uncertainties on the inclination. We compute an inclination $i=61^{\circ} \pm 5^{\circ}$. The dynamical mass is defined by Equation (3),

$$
M_{\text {dyn }}=\frac{R V^{2}}{G},
$$

where $V=V_{r} \sin (i) \quad\left(V_{r}\right.$ is the radial velocity, $i$ is the inclination) and $R$ is the radius. Förster Schreiber et al. (2009) uses the velocity at $10 \mathrm{kpc}$, which is typically the surface brightness limit of their sample, to determine the dark matter contribution of their galaxies. Looking at the rotation curves of nearby galaxies (Sofue et al. 1999), many of the rotation curves flatten out well beyond $10 \mathrm{kpc}$, which means that the baryons contribute more to the inner $10 \mathrm{kpc}$ than dark matter. For AS1063a, the rotation curve flattens out after $4-5 \mathrm{kpc}$, and it is not unreasonable to extrapolate the velocity at $10 \mathrm{kpc}$. Courteau (1997) demonstrates that the arctangent function is a good fit to galaxy rotation curves in the local universe in order to determine the circular velocity at a given radius, shown in Equation (4):

$$
V(R)=V_{0}+\frac{2}{\pi} V_{c} \arctan \frac{R}{R_{t}},
$$

Table 7

Metallicity of AS1063a

\begin{tabular}{lcc}
\hline \hline & $12+\underset{\mathrm{R} 23}{\log ([\mathrm{O} / \mathrm{H}])}$ & $12+\underset{\mathrm{N} 2}{\log ([\mathrm{O} / \mathrm{H}]})$ \\
\hline Entire galaxy & $8.95 \pm 0.02$ & $8.80 \pm 0.03$ \\
H II region & $8.96 \pm 0.02$ & $\cdots$ \\
Bulge & $8.99 \pm 0.02$ & $\cdots$ \\
Spiral arm & $8.74 \pm 0.10$ & $\cdots$ \\
\hline
\end{tabular}

where $V_{0}$ is the velocity center of rotation (systemic velocity), $V_{c}$ is the asymptotic velocity, and $R_{t}$ is the scale radius where the rotation curve begins to flatten out. We fit the AS1063a velocity curve with the arctangent function. Figure 12 (right panel) shows the fit to the intrinsic radius. The large error bars and scatter seen in the points that are furthest from the center are due to the low $\mathrm{S} / \mathrm{N}$ of the fit to the [O II] line. If you allow the [O II] line to be fit at larger radius, the error bar increases significantly, which once again reflects the $\mathrm{S} / \mathrm{N}$ decreasing.

With the inclination and the circular velocity at $10 \mathrm{kpc}$, we compute a dynamical mass of $(5 \pm 1) \times 10^{10} \mathrm{M}_{\odot}$. This dynamical mass is comparable to the average Spectroscopic Imaging survey in the Near-IR with SINFONI (SINS; Förster Schreiber et al. 2009) galaxy, which spans $\sim(1-20) \times 10^{10} \mathrm{M}_{\odot}$ for redshifts $z=1.3-2.6$.

\subsubsection{Metallicity}

As mentioned in Section 3.2 we detect key emission lines with LDSS-3 and MMIRS for determining metallicity. We use both R23 ([O II] $\lambda 3727, \mathrm{H} \beta$, and [O III] $\lambda 5007$ ) and [O III]/[O II] and $\mathrm{N} 2(\mathrm{H} \alpha$ and $[\mathrm{N}$ II] $\lambda 6585)$ diagnostics to determine the metallicity of the lensed galaxy at $z=0.61$ and its individual regions. We were unable to detect the $[\mathrm{O}$ III $] \lambda 4363$ line, a lowmetallicity indicator, which implies that we should choose the upper branch for R23.

For the N2 measurement we follow the prescription of Pettini \& Pagel (2004) using their cubic relation. For the R23 measurement, we follow the prescription of Kewley \& Ellison (2008), specifically following the KK04 method. The results for the metallicities can be found in Table 7. All the values are roughly consistent with solar metallicity. There is some evidence of a metallicity gradient from the spiral arm to the bulge. The metallicity is constant from the bulge to the H II region.

Metallicity gradients are seen in the Milky Way, where the gas-phase metallicity is lower at larger radii than at the bulge. These metallicity gradients are also seen in local galaxies, where the slope of the gradient is shallow. The gradient in metallicity suggests that the metallicity throughout the disk of a galaxy is evolving over time. At higher redshift, in $z \sim 2$ galaxies (Jones et al. 2010a, 2013; Yuan et al. 2011), there is evidence for steeper metallicity gradients. It is suggested that there is inside-out growth in these galaxies that may be responsible for creating the steep metallicity gradients. In AS1063a, the metallicity gradient is not as steep as seen in $z \sim 2$ galaxies and appears more like galaxies in the local universe, shown in Figure 13.

There are known aperture effects when measuring metallicity, where low-metallicity regions may be hidden by higher metallicity regions. While it appears that the metallicity is constant between the H II region and the bulge of AS1063a, we 


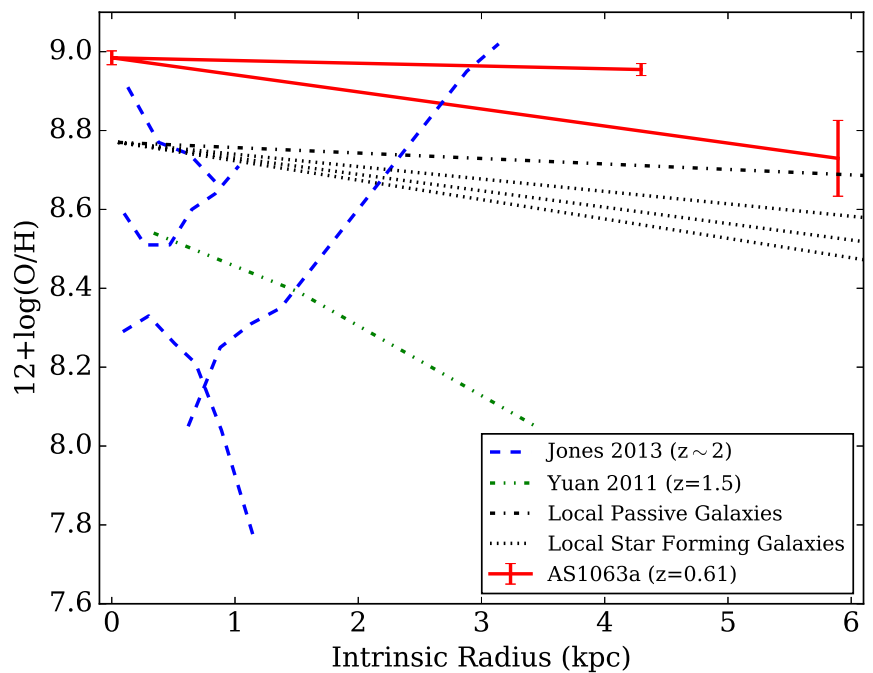

Figure 13. Metallicity as a function of radius for galaxies in the local universe (Henry \& Worthey 1999; Rupke et al. 2010) and at a high-redshift gravitationally lensed sample (Yuan et al. 2011; Jones et al. 2013). The higher redshift galaxies show a steeper metallicity gradient than is seen in the local universe. AS1063a resembles a more local star-forming galaxy. We use the R23 to derive the metallicity in AS1063a. Jones et al. (2013) uses a variety of methods; we compare to their O3HB. Yuan et al. (2011) uses N2.

may not be sensitive to the lower metallicity regions. This is primarily due to the spatial resolution of the spectra, determined by the seeing, and $\mathrm{S} / \mathrm{N}$ of the fainter emission lines, such as the $[\mathrm{O}$ III $] \lambda 4959$.

The Spitzer Infrared Nearby Galaxies Survey (SINGS) sample of H II regions (Moustakas et al. 2010) in the local universe spans metallicities 7.7-9.3 (KK04). The majority of $\mathrm{H}$ II regions $(88 \%)$ have metallicities between 8.6 and 9.2. The metallicity of the HII region in AS1063a does not seem unusual when compared to the SINGS galaxies in the local universe as $48 \%$ of their $\mathrm{H}$ II regions have metallicities between 8.9 and 9.1. Higher redshift $\mathrm{H}$ II regions seem to have lower metallicities when compared with the H II region in AS1063a. Wisnioski et al. (2012) metallicities span 8.4-8.8, Jones et al. (2010a, 2013) span 8.2-9.0. and Wuyts et al. (2014) span 8.0-8.3 for several H II regions in RCSGA0327.

\subsubsection{Gas Depletion Timescale}

In order to estimate the molecular gas mass without a $\mathrm{CO}$ measurement there are two methods: (1) using the dust mass from the galaxy's far-IR emission and (2) using the dynamical mass from the galaxy's kinematics.

For the first method in determining gas mass, we need to measure the dust mass. To determine the dust mass from the far-IR emission, we use Equation (5) (Greve et al. 2012),

$$
M_{\text {dust }}=\frac{D_{L}^{2} S_{\nu_{o}}}{(1+z) \kappa_{\nu_{r}}}\left[B_{\nu_{r}}\left(T_{\text {dust }}\right)-B_{\nu_{r}}\left(T_{\mathrm{CMB}}(z)\right)\right]^{-1},
$$

where $S_{\nu_{o}}$ is the observed flux, $B_{\nu_{r}}$ is the rest-frame Planck function, and $\kappa_{\nu_{r}}$ is the rest-frame absorption coefficient. The Planck function is defined at the dust temperature $T_{\text {dust }}$ and the $T_{\mathrm{CMB}}$ at redshift $z$. The absorption coefficient is defined as $\kappa_{\nu_{r}}=0.45\left(\nu_{r} / 250 \mathrm{GHz}\right)^{\beta} \quad$ (Hildebrand 1983; Kruegel \& Siebenmorgen 1994), where $\nu_{r}$ is the rest-frame frequency and $\beta=2.0$. According to Papadopoulos et al. (2000) the temperature of the cosmic microwave background (CMB) affects the dust mass by $\sim 2 \%$. da Cunha et al. (2013) also found that at redshifts $z<4$ the $\mathrm{CMB}$ temperature negligibly affects the dust mass, and because of this we do not include it in our calculation. Using a $T_{\text {dust }}=36 \pm 1 \mathrm{~K}$ and using the LABOCA $870 \mu \mathrm{m}$ flux we compute $M_{\text {dust }}=(4.3 \pm 0.4) \times 10^{7} \mathrm{M}_{\odot}$.

In order to compute the gas mass from the dust mass it is important to know the dust-to-gas ratio (DGR). Leroy et al. (2011) and Sandstrom et al. (2013) show that there is a correlation between metallicity and the DGR for local starforming galaxies and parameterized it using Equation (6) for metallicities computed using KK04:

$$
\log (\mathrm{DGR})=a+b(12+\log (\mathrm{O} / \mathrm{H})-c),
$$

where $a=-1.86, \quad b=0.85$, and $c=8.39$. Using the metallicity found for the entire galaxy from the R23 line ratio we find a DGR $=0.0114$.

The relationship between molecular gas, $\mathrm{HI}$, and dust is shown in Equation (7) (Leroy et al. 2011; Sandstrom et al. 2013):

$$
\Sigma_{\mathrm{D}} / \mathrm{DGR}=\Sigma_{\mathrm{HI}}+\Sigma_{\mathrm{H}_{2}},
$$

where $\Sigma_{\mathrm{D}}$ is the mass surface density of dust, $\Sigma_{\mathrm{H} \text { I }}$ is the mass surface density of $\mathrm{HI}$, and $\Sigma_{\mathrm{H}_{2}}$ is the mass surface density of molecular gas. $\mathrm{H}$ I is difficult to detect at higher redshift (e.g., $z=0.4$ galaxy observed for $180 \mathrm{hr}$ with the Very Large Array, Fernández et al. 2016), and because of this it is typically excluded from the total gas mass. However, LIRGs and ULIRGs (ultraluminous IR galaxies, $L_{\mathrm{IR}} \geqslant 10^{12} \mathrm{~L}_{\odot}$ ) are observed in the local universe to have more molecular gas than atomic (e.g., $\mathrm{H}_{2} / \mathrm{HI}>1$, Mirabel \& Sanders 1989), with the ratio of $\mathrm{H}_{2} / \mathrm{H}$ I increasing with IR luminosity. At most, the total molecular gas could be off by about $0.3 \mathrm{dex}$. More typically for submillimeter galaxies and ULIRGS the total H I mass is not significant (0.1 dex, Sanders \& Mirabel 1996; Santini et al. 2010). We find that the molecular gas mass is the following: $M_{\text {gas }}=(3.8 \pm 0.4) \times 10^{9} \mathrm{M}_{\odot}$.

With the gas mass and far-IR SFR $\left(54 \pm 2 \mathrm{M}_{\odot} \mathrm{yr}^{-1}\right)$, we compute the gas depletion timescale of $70 \mathrm{Myr}$. If instead we assume a more conservative SFR, from the instantaneous SFR derived from $\mathrm{H} \alpha\left(33 \pm 1 \mathrm{M}_{\odot} \mathrm{yr}^{-1}\right)$, it would suggest a depletion timescale of $120 \mathrm{Myr}$.

Comparing the gas mass to the stellar mass gives the gas fraction $f_{\mathrm{gas}}=0.13_{-0.04}^{+0.06}$, defined as $\left.f_{\mathrm{gas}}=M_{\mathrm{gas}} /\left(M_{\mathrm{gas}}+M_{*}\right)\right)$, or $M_{\text {gas }}=(4.1 \pm 0.4) \times 10^{9} \mathrm{M}_{\odot}$. Comparing to other galaxies at this redshift (Daddi et al. 2010; Geach et al. 2011; Magdis et al. 2012), the gas fraction for this galaxy is typical and not unusual. The range of gas fractions at $z \sim 0.6$ is about $0.05-0.3$.

For the second method of determining the gas mass, we use the dynamical mass of the galaxy from Section 4.2.4. The gas mass is defined as $M_{\mathrm{gas}}=M_{\mathrm{dyn}}-M_{*}-M_{\mathrm{dark}}$, where the $M_{\text {dark }}$ is the dark matter mass. If we assume a dark matter fraction of 0.2-0.3 within a radius of $10 \mathrm{kpc}$ (Förster Schreiber et al. 2009) for galaxies $z=1-3$ we get a gas mass of $M_{\text {gas }}=(7.7-12.4) \times 10^{9} \mathrm{M}_{\odot}, \quad$ which $\quad$ is $f_{\text {gas }}=0.23-0.33$, which is larger than what we compute using the dust mass. There are likely more uncertainties with the dynamical mass calculation, as the dynamical mass could be influenced by the position of the slit, not fully sampling the galaxy's velocity field. In addition, inclination of the galaxy could also be a large uncertainty, as the projection of the galaxy on the sky is highly 
Table 8

Physical Properties of AS1063a

\begin{tabular}{lcc}
\hline \hline Parameter & Value & Unit \\
\hline$T$ & $36 \pm 1$ & $\mathrm{~K}$ \\
$L_{\text {IR }}$ & $(3.1 \pm 0.1) \times 10^{11}$ & $\mathrm{~L}_{\odot}$ \\
SFR FIR & $54 \pm 2$ & $\mathrm{M}_{\odot} \mathrm{yr}^{-1}$ \\
SFR H $\alpha$ & $33 \pm 1$ & $\mathrm{M}_{\odot} \mathrm{yr}^{-1}$ \\
$M_{\text {dust }}$ & $(4.6 \pm 0.4) \times 10^{7}$ & $\mathrm{M}_{\odot}$ \\
$M_{*}$ & $2.57_{-0.95}^{+1.50} \times 10^{10}$ & $\mathrm{M}_{\odot}$ \\
$M_{\text {dyn }}$ & $(5 \pm 1) \times 10^{10}$ & $\mathrm{M}_{\odot}$ \\
$M_{\text {gas }}$ & $(4.1 \pm 0.4) \times 10^{9}$ & $\mathrm{M}_{\odot}$ \\
$E(B-V)$ & $0.36 \pm 0.04$ & \\
$A_{\mathrm{V}}$ & $1.5 \pm 0.2$ & $\mathrm{mag}$ \\
$f_{\text {gas }}\left(M_{\text {dust }}\right)$ & $0.13_{-0.04}^{+0.06}$ & \\
$i$ & $61 \pm 5$ & $\mathrm{deg}$ \\
\hline
\end{tabular}

degenerate, especially when considering diverse galaxy morphologies. Finally, the stellar mass could be underestimated, which could result in a larger gas fraction. From here on, we use the gas fraction based on the dust mass. A summary of the physical properties can be found in Table 8 .

In Section 4.2.2 we find that as much as half of the far-IR flux could be associated with the H II region (half of the SFR). Extending this to the molecular gas, if we assume that the molecular gas traces the dust, then roughly half of the molecular gas could be associated with the $\mathrm{H}$ II region. This also suggests a similar depletion timescale of $70 \mathrm{Myr}$. Using the instantaneous SFR derived from $\mathrm{H} \alpha$ from the $H S T$ grism $\left(8 \mathrm{M}_{\odot} \mathrm{yr}^{-1}\right)$ for the H II region would suggest a depletion timescale of $230 \mathrm{Myr}$. Adding the depletion timescale to the stellar age $\left(0.38_{-0.17}^{+0.31} \mathrm{Gyr}\right)$ of the AS1063a $\mathrm{H}$ II region makes its lifetime 0.44-0.92 Gyr. However, even with a Balmer decrement, it is unclear whether we are truly measuring the current instantaneous SFR or the dust-attenuated one. In order to determine the lifetime of the $\mathrm{HII}$ region, even without instantaneous star formation tracers that are less affected by dust (i.e., $\mathrm{P} \alpha, \mathrm{P} \beta$ ), we rely on the far-IR SFR to set the maximum rate at which stars form. We find that incorporating the range of SFRs and stellar ages provides a lifetime for the $\mathrm{H}$ II region being 0.28 and $0.92 \mathrm{Gyr}$. This is consistent with $\mathrm{H}$ II regions at $z \sim 2$, which are expected to live between 0.1 and 1 Gyr (Dekel et al. 2009; Genzel et al. 2011; Guo et al. 2012).

\subsection{Giant ( $\sim k p c)$ H II Region Embedded in a Rotating Disk at $\mathrm{z}=0.61$}

Taking into account the effect of magnification, we divide by the linear magnification, determined by the lens model of the cluster, and find the FWHM of the clump in the source plane is $996 \pm 46 \mathrm{pc}$. In order to compute the error bar for the size of the H II region we ran a Monte Carlo simulation, varying the noise and refitting the elliptical Gaussian for 1000 realizations, then measuring the standard deviation of the width measurements.

At the spatial resolution provided by the gravitational lensing, this means that the clump appears to be a single clump, with ACS resolving individual structures $>90 \mathrm{pc}$ and WFC3/IR $>240$ pc. While we cannot completely rule out the possibility, it appears unlikely to have $>10$ smaller clumps lined up in a chain, appearing like a single $1 \mathrm{kpc}$ clump.
4.3.1. As a Low-redshift Analog to Giant H II Regions at $\mathrm{z} \gtrsim 1$

Figure 14 shows the SFR of individual clumps versus their radius for nearby galaxies and galaxies with redshifts $z>0.8$. When plotted on this relation, the H II region of the $z=0.61$ lensed galaxy found in AS1063 is more luminous than typical local clumps (by $\sim 2$ orders of magnitude) and as large as the largest clumps found locally. This $\mathrm{H}$ II region is almost a factor of 10 larger in radius than the mean radius of local clumps. When comparing to high-redshift $(z>0.8)$ clumps, the H II region is similar in size and luminosity. What is particularly striking about this star-forming clump is how it resembles clumps at the highest redshifts. It appears more similar in size and luminosity to the clumps at $z>3.0$ (and the more luminous clumps $1.5<z<3.0$ ).

Local clumps, on average, are much smaller $(\sim 50-200 \mathrm{pc}$ in radius) than high-redshift $(z>0.8)$ clumps and less luminous (SFR $\left.\sim 0.0001-0.01 \mathrm{M}_{\odot} \mathrm{yr}^{-1}\right)$. However, there are exceptional cases of local galaxies with large star-forming regions almost spanning the size range for higher redshift clumps, but at lower luminosity. For comparison, one of the largest local H II regions is overplotted in Figure 14, NGC 604 in M33.

Local star-forming galaxies (from the SINGS sample) appear to fall on a surface brightness empirical relation, where as a clump becomes brighter, it also gets larger. It has been suggested that this relation evolves with redshift in galaxies along the "main sequence," where higher redshift clumps are more luminous than lower redshift clumps for a given size (Livermore et al. 2012, 2015). This evolution may be driven by a galaxy's gas fraction where the higher redshift galaxies are found to have higher gas fractions than the local galaxies. However, Mieda et al. (2016) found large resolved clumps (using an integral field spectrograph) in high-redshift $(z \sim 1)$ galaxies, with a wide range of SFRs, which appear to be scaledup versions of local H II regions. Additionally, Johnson et al. (2017a) find that a $z=2.48$ galaxy with a low SFR $\left(9 \mathrm{M}_{\odot} \mathrm{yr}^{-1}\right)$ contains star-forming clumps that are $30-50 \mathrm{pc}$ in size. This hints that there is another process at work. Cosens et al. (2018) recently suggested that there is no redshift evolution in the current clump samples and that there are really two populations of star-forming clumps. The populations can be divided based on whether or not a galaxy is undergoing a starburst (i.e., $\Sigma_{\mathrm{SFR}}$ ).

Another key feature is that the clump in AS1063a appears to have formed in isolation (in situ). As we show in Section 4.2.4 the clump appears to be embedded in a rotating disk with no evidence of interaction. This is typical of the clumps found at high redshift $(z>2)$ but uncommon for local galaxies. For galaxies at low redshift it is found that massive luminous starforming regions are induced by the interacting galaxies. It is suggested that the conditions at higher redshift, higher gas fractions and cold flow accretion, may be responsible for clumps forming in isolation, perhaps due to gravitational instability. They could also be short-lived features, as work from Dekel et al. (2009), Genzel et al. (2011), and Guo et al. (2012) has suggested that clumps can migrate or diffuse within 0.1-1 Gyr. We also know that $\mathrm{H} \alpha$ emission traces recent star formation (Kennicutt \& Evans 2012) within the last 3-10 Myr.

It would be expected that such a clump at this redshift would be quite rare. However, two of the largest and brightest clumps in the Livermore et al. (2012) sample $(z=1-1.5)$ are at redshift $z=1.01$ (Abell 773). These clumps are more comparable to the size and luminosity of clumps in the 


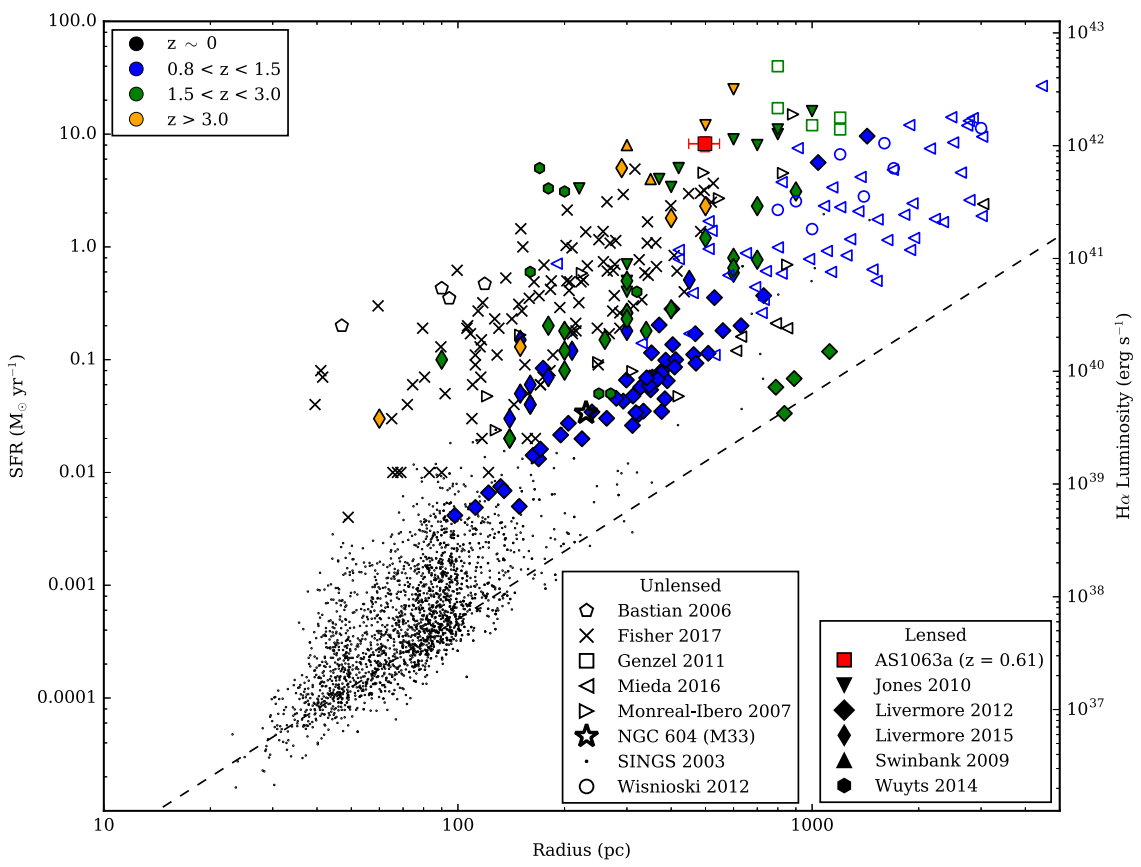

Figure 14. Relation between SFR and the radius of H II regions (adapted from Livermore et al. 2012; Wisnioski et al. 2012). The red point is the H II region from AS1063a at $z=0.61$. The other points are all galaxies above $z>0.8$, color coded by redshift, by combining samples of lensed (solid color symbols; Swinbank et al. 2009; Jones et al. 2010b; Livermore et al. 2012, 2015; Wuyts et al. 2014) and unlensed (open symbols; Förster Schreiber et al. 2011b; Wisnioski et al. 2012; Mieda et al. 2016) galaxies. The gray points are from SINGS galaxies (Kennicutt et al. 2003). The open black symbols are giant $\mathrm{H}$ II regions found at $z \sim 0$ in Antennae galaxies (Bastian et al. 2006) and ULIRGs (Monreal-Ibero et al. 2007). The black star is the giant H II region NGC 604 found in M33 (Relaño \& Kennicutt 2009 ). The black x's are large star-forming clumps from nearby galaxies (Fisher et al. 2017). The dashed line represents a line of constant H $\alpha$ surface brightness, which is proportional to $R^{2}$.

Jones et al. (2010b) sample at redshift $z \sim 2$ and the Wisnioski et al. (2012) sample (WiggleZ). Recent work by Guo et al. (2015) has shown that a galaxy's stellar mass determines the frequency of clumps found from redshifts $z=0.5-2$. The clump fraction remains constant for galaxies with a smaller stellar mass, whereas the clump fraction decreases with time over the redshift range for higher stellar mass galaxies.

\section{Conclusions}

In this paper we present the results of the IR/submillimeter survey of the core of the massive galaxy cluster AS1063. Three bright $24 \mu \mathrm{m}$ detected sources near $\left(r<30^{\prime \prime}\right)$ the cluster core of AS1063 stand out in the survey. Two of the sources are cluster members (AS1063b and AS1063c) with recent star formation. The third source is a lensed galaxy at $z=0.61$ (AS1063a). We also present evidence that AS1063a contains a giant $\mathrm{H}$ II region that is about a kiloparsec in diameter and 2 orders of magnitude more luminous than typical local H II regions, with strong Balmer line emission ( $\mathrm{H} \alpha-\mathrm{H} 8)$ and forbidden line emission ([O II] $\lambda 3727$, [O III] $\lambda \lambda 4959,5007$, and [Ne III]).

The main conclusions in this paper are the following:

1. We discover a gravitationally lensed galaxy (AS1063a) with a giant luminous $\mathrm{H}$ II region $(\mathrm{D}=1 \mathrm{kpc}$, $\mathrm{SFR} \sim 10 \mathrm{M}_{\odot} \mathrm{yr}^{-1}$ ) at $z=0.61$. It appears to be a single clump with no additional clumps resolved within it larger than $90 \mathrm{pc}$ (ACS resolution with the aide of gravitational lensing).

2. The kinematics of the $[\mathrm{O}$ II $] \lambda 3727$ doublet indicate that the giant $\mathrm{H}$ II region is part of a rotating disk. There is no evidence of any nearby galaxies interacting with AS1063a, which suggests that this H II region formed in situ.
3. We find a significant offset between the $24 \mu \mathrm{m}$ emission and center of AS1063a, in which it falls between the bulge and $\mathrm{H}$ II region of the galaxy. When fitting the extended $24 \mu \mathrm{m}$ emission, we find that it is likely that half of the star formation in AS1063a is coming from the giant $\mathrm{H}$ II region, although we cannot rule out the possibility of an obscured star-forming region, due to the degeneracy of the fits to the MIPS PSF.

4. Using both a DGR and kinematics of the galaxy rotation we are able to determine the gas fraction, $f_{\text {gas }}=0.13_{-0.04}^{+0.06}$, which is typical for LIRGs at that redshift. At $z \sim 2$ gas fractions are normally $0.3-0.8$.

5. Assuming that about half of the star formation comes from the $\mathrm{H}$ II region and using the gas fraction imply that the depletion timescale for the H II region is roughly between 70 and $230 \mathrm{Myr}$. Incorporating the stellar age, the lifetime of the H II is predicted to be 280-920 Myr, which makes it short-lived.

The H II region in AS1063a is more luminous than local analogs and is unexpectedly luminous for its redshift, resembling an $\mathrm{H}$ II region at $z \sim 2$. This could potentially be a rare occurrence; however, in the Livermore et al. (2012) sample, two star-forming clumps found in a lensed galaxy in Abell 773 have similar luminosity and size at $z=1.01$. In addition, the giant $\mathrm{H}$ II region in AS1063a appears to have formed in isolation and to have been not induced by a merger. Unlike giant star-forming regions in the local universe, the giant $\mathrm{H}$ II region in AS1063a appears more like the ones found in redshift $z \sim 2$ galaxies. Even though recent studies (Guo et al. 2015) have determined the fraction of clumps in field galaxies at redshifts $z=0.5-2$, they are typically unresolved. Larger samples of gravitationally lensed IR galaxies will be 
necessary to determine the distribution of sizes and luminosities of resolved star-forming regions.

G.L.W. thanks Dan Kelson, Patrick Sheehan, Justin Spilker, Decker French, Andrey Vayner, Cliff Johnson, Maren Cosens, Thomas Connor, Brenda Frye, Shelley Wright, George Rieke, Xiaohui Fan, Dennis Zaritsky, and Dan Marrone for useful discussions.

P.G.P.-G. acknowledges support from Spanish Government MINECO AYA2015-70815-ERC and AYA2015-63650-P Grants.

Facilities: APEX (LABOCA), Herschel Space Observatory (PACS, SPIRE), Hubble Space Telescope (ACS, WFC3), Magellan:Clay (LDSS-3, MMIRS), Spitzer Space Telescope (IRAC, IRS, MIPS).

Software: aXe (Kümmel et al. 2009), COSMOS (Dressler et al. 2011), GALFIT (Peng et al. 2002, 2010), IRAF (Tody 1986, 1993), LENSTOOL (Jullo et al. 2007), PAHFIT (Smith et al. 2007), SExtractor (Bertin \& Arnouts 1996), TinyTim (Krist et al. 2011), UniMap (Piazzo et al. 2015).

\section{Appendix \\ $24 \boldsymbol{\mu m}$ Selected Galaxies with Spectroscopic Redshifts}

In Tables 9 and 10 we list the 71 Spitzer/MIPS $24 \mu \mathrm{m}$ sources in this sample, 61 of which were targeted with Magellan/LDSS-3, and 10 of which have redshifts from the literature: Gómez et al. (2012), Karman et al. (2015, 2017), Caminha et al. (2016), Rawle et al. (2016), Connor et al. (2017). Spectroscopic redshifts for all of the Spitzer/MIPS $24 \mu \mathrm{m}$ counterparts are listed in Table 9. The Spitzer/MIPS $24 \mu \mathrm{m}$ sources targeted with LDSS-3 but with a spectroscopic redshift that could not be determined are listed in Table 10. For the sources targeted with LDSS-3, a spectroscopic redshift was determined with either one or more emission or absorption features. The redshifts for $2324 \mu \mathrm{m}$ sources were previously unknown, mostly between the redshifts $0.6<z<1.4$. The emission lines detected are listed in Table 9 for the LDSS-3 sample. We adopt similar spectroscopic quality codes as the DEEP2 survey. The redshift quality codes are defined as the following: for a $z_{q}$ of 2 there is a single emission or absorption feature where the redshift is dubious, for a $z_{q}$ of 3 there are few emission or absorption features where the redshift is secure, and for a $z_{q}$ of 4 there are several emission and absorption features and the redshift is very secure.

Figure 15 shows the positions of the galaxies from our sample and the literature. From the spectroscopic sample, we find that 29 galaxies are in the background, 15 are cluster members, and five are foreground galaxies. For one of the sources, the correct counterpart is ambiguous; there are two galaxies within 1!"58 of each other, and they both fall within the LDSS-3 slit. Based on the distance from the optical counterparts to the $24 \mu \mathrm{m}$ source, the lower redshift galaxy $(z=0.474)$ appears to be the more likely candidate for the $24 \mu \mathrm{m}$ emission. Of the 29 background galaxies, 12 have redshifts $z>1$, one of which is within the central arcminutes of the cluster center. Of the 24 galaxies with previously unknown redshifts, 20 of the galaxies are found in the background of the cluster.

We also identify five galaxies at redshift $z \sim 0.6$, one of which is discovered by Gómez et al. (2012). The spatial distances between these galaxies and AS1063a, when accounting for the deflection caused by gravitational lensing, are 230, 470, 580, and $980 \mathrm{kpc}$. This study, Gómez et al. (2012), and Karman et al. (2015) identify six galaxies at $z \sim 0.6$, all within $2 ! 4$ of each other and a $\Delta v$ of $2400 \mathrm{~km} \mathrm{~s}^{-1}$ (five within 1!.4 and with $\Delta v$ of $370 \mathrm{~km} \mathrm{~s}^{-1}$ ). Gruen et al. (2013) uses weak lensing to suggest that there is a background cluster at $z \sim 0.6$, offset by 14.5 from AS1063. This could potentially be an infalling group; however, this is speculative. More wide-field spectroscopy is needed to determine the size and structure of the suspected background cluster at $z \sim 0.6$. 
Table 9

Spectroscopic Redshifts of $24 \mu \mathrm{m}$ Selected Galaxies

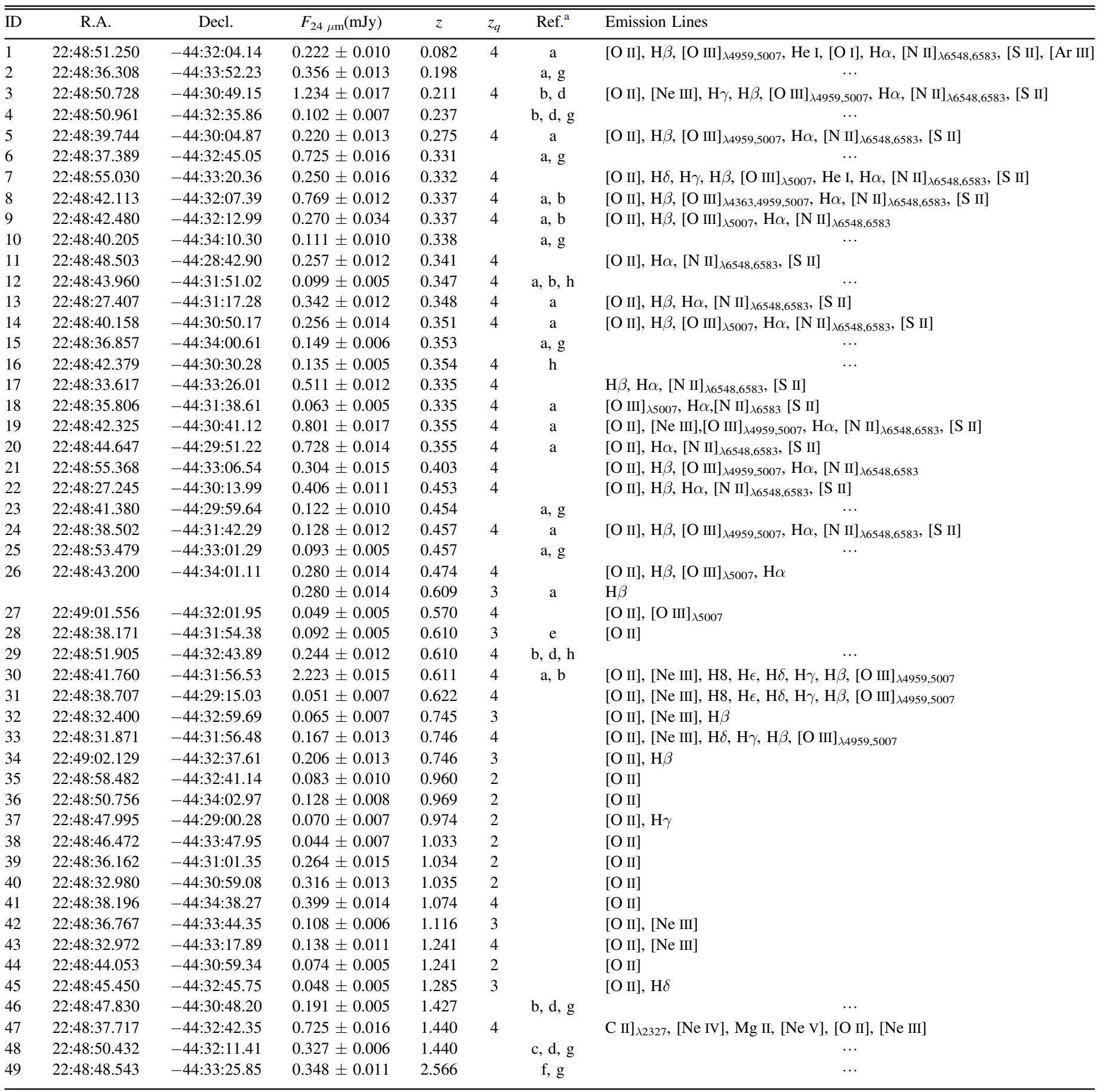

Notes. Emission lines in the table are abbreviations of the following: [Ar III] $-[\mathrm{Ar}$ III] $] \lambda 7135$, He I-He I $\lambda 5876, \mathrm{Mg}$ II $-\mathrm{Mg}$ II $\lambda 2796,2804$, [Ne III] $-[\mathrm{Ne}$ III] $\lambda 3869$,

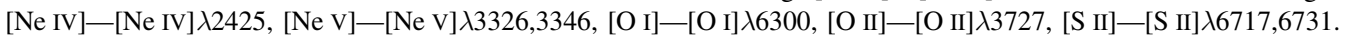

a (a) Spectroscopic redshift from Gómez et al. (2012), (b) spectroscopic redshift from Karman et al. (2015, 2017); Caminha et al. (2016), (c) spectroscopic redshift from Rawle et al. (2016), (d) spectroscopic redshift from P. Rosati et al. (2019, in preparation), (e) spectroscopic redshift reported in P. Rosati et al. (2019, in preparation) but not Gómez et al. (2012) catalog, (f) spectroscopic redshift from Connor et al. (2017), (g) source not targeted with LDSS-3, (h) no emission lines detected, redshift determined with absorption features. 


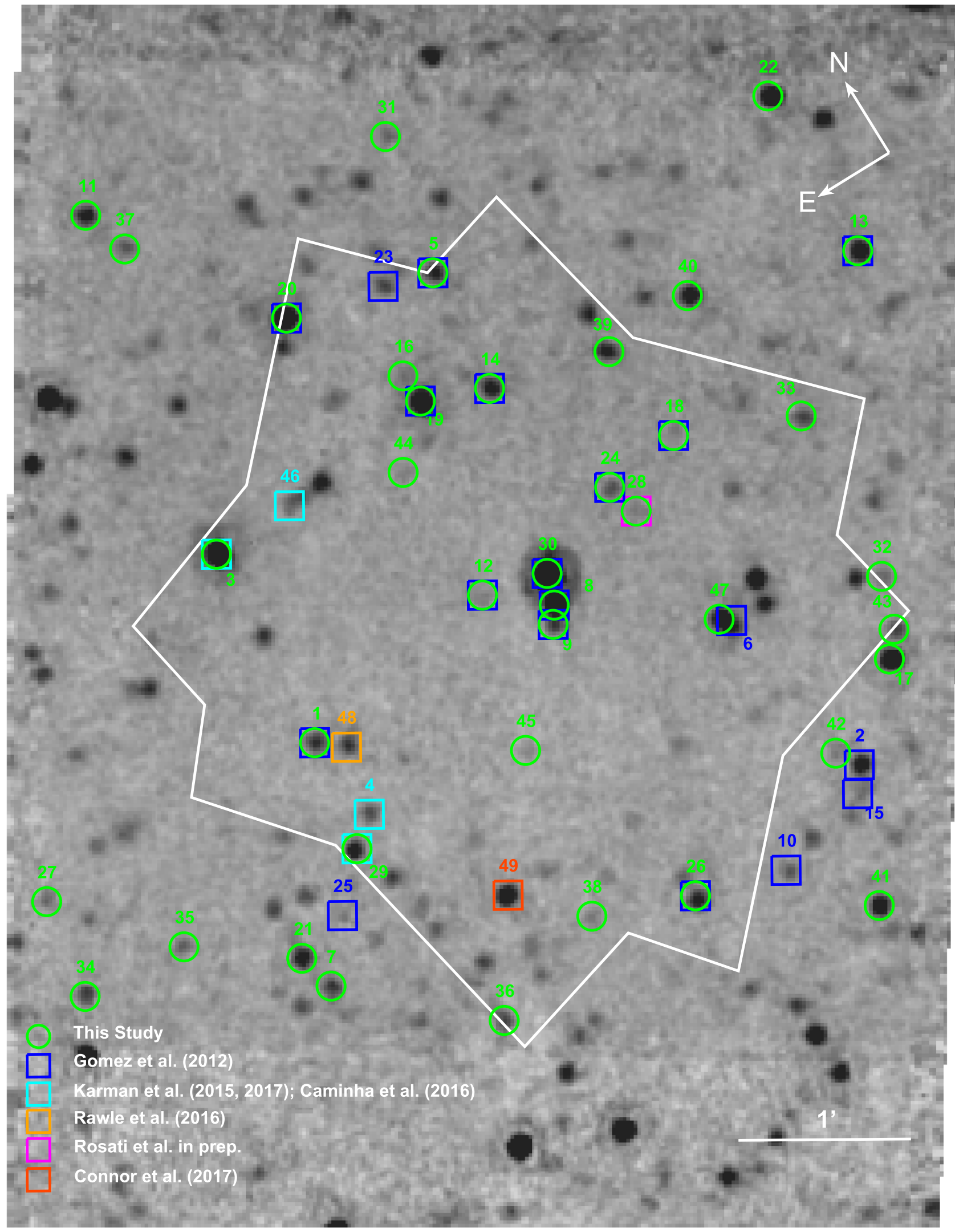

Figure 15. Spitzer/MIPS $24 \mu \mathrm{m}$ image showing the central $5^{\prime} \times 5^{\prime}$ FOV of AS1063. All of the $24 \mu \mathrm{m}$ sources that are marked have a redshift. The green circles mark the positions of $24 \mu \mathrm{m}$ selected galaxies targeted by this study. The squares mark the positions of the galaxies with redshifts from Gómez et al. (2012), Karman et al. (2015), Caminha et al. (2016), Rawle et al. (2016), Connor et al. (2017), Karman et al. (2017), and P. Rosati et al. (2019, in preparation), which are now known to have $24 \mu \mathrm{m}$ emission. Source 30 is AS1063a (the main focus of this paper), source 8 is AS1063b, and source 9 is AS1063c. The white polygon represents the CLASH ACS FOV. 
Table 10

$24 \mu \mathrm{m}$ Selected Galaxies Targeted without a Redshift

\begin{tabular}{lccc}
\hline \hline ID & R.A. & Decl. & $F_{24 \mu \mathrm{m}}(\mathrm{mJy})$ \\
\hline 50 & $22: 48: 38.351$ & $-44: 29: 43.46$ & $0.146 \pm 0.011$ \\
51 & $22: 48: 33.829$ & $-44: 30: 30.27$ & $0.083 \pm 0.009$ \\
52 & $22: 48: 38.390$ & $-44: 33: 17.88$ & $0.089 \pm 0.006$ \\
53 & $22: 48: 51.484$ & $-44: 32: 59.22$ & $0.153 \pm 0.012$ \\
54 & $22: 48: 35.514$ & $-44: 27: 54.21$ & $0.232 \pm 0.014$ \\
55 & $22: 48: 52.765$ & $-44: 29: 30.57$ & $0.650 \pm 0.011$ \\
56 & $22: 48: 36.662$ & $-44: 34: 19.83$ & $0.072 \pm 0.009$ \\
57 & $22: 48: 38.959$ & $-44: 34: 05.20$ & $0.121 \pm 0.010$ \\
58 & $22: 48: 37.595$ & $-44: 34: 54.06$ & $0.234 \pm 0.013$ \\
59 & $22: 48: 26.323$ & $-44: 30: 46.24$ & $0.077 \pm 0.005$ \\
60 & $22: 48: 33.278$ & $-44: 30: 05.67$ & $0.082 \pm 0.008$ \\
61 & $22: 48: 31.255$ & $-44: 30: 19.07$ & $0.085 \pm 0.007$ \\
62 & $22: 48: 29.689$ & $-44: 30: 35.04$ & $0.030 \pm 0.005$ \\
63 & $22: 48: 36.515$ & $-44: 30: 14.18$ & $0.073 \pm 0.009$ \\
64 & $22: 48: 34.301$ & $-44: 30: 51.34$ & $0.127 \pm 0.012$ \\
65 & $22: 48: 40.691$ & $-44: 30: 41.67$ & $0.094 \pm 0.006$ \\
66 & $22: 48: 46.584$ & $-44: 30: 46.90$ & $0.249 \pm 0.009$ \\
67 & $22: 48: 36.050$ & $-44: 32: 36.58$ & $0.432 \pm 0.012$ \\
68 & $22: 48: 50.861$ & $-44: 31: 22.54$ & $0.130 \pm 0.007$ \\
69 & $22: 48: 53.780$ & $-44: 32: 26.19$ & $0.087 \pm 0.009$ \\
70 & $22: 49: 03.205$ & $-44: 32: 54.00$ & $0.439 \pm 0.011$ \\
71 & $22: 48: 23.267$ & $-44: 30: 38.31$ & $0.078 \pm 0.006$ \\
\hline & & &
\end{tabular}

\section{ORCID iDs}

Gregory L. Walth (iD https://orcid.org/0000-0002-6313-6808 Johan Richard (1) https://orcid.org/0000-0001-5492-1049 Miroslava Dessauges-Zavadsky (i) https://orcid.org/00000003-0348-2917

Benjamin Weiner (D) https://orcid.org/0000-0001-6065-7483

Ian McGreer (ib https://orcid.org/0000-0002-3461-5228

Evan Schneider (iD https://orcid.org/0000-0001-9735-7484

\section{References}

Armus, L., Heckman, T. M., \& Miley, G. K. 1990, ApJ, 364, 471 Baldwin, J. A., Phillips, M. M., \& Terlevich, R. 1981, PASP, 93, 5 Balestra, I., Vanzella, E., Rosati, P., et al. 2013, A\&A, 559, L9 Barnes, E. I., \& Sellwood, J. A. 2003, AJ, 125, 1164

Barnes, J. E., \& Hernquist, L. E. 1991, ApJL, 370, L65 Bastian, N., Emsellem, E., Kissler-Patig, M., \& Maraston, C. 2006, A\&A, 445, 471 Bertin, E., \& Arnouts, S. 1996, A\&AS, 117, 393

Boone, F., Clément, B., Richard, J., et al. 2013, A\&A, 559, L1

Bournaud, F., Elmegreen, B. G., \& Elmegreen, D. M. 2007, ApJ, 670, 237 Bruzual, G., \& Charlot, S. 2003, MNRAS, 344, 1000

Caminha, G. B., Grillo, C., Rosati, P., et al. 2016, A\&A, 587, A80

Cava, A., Schaerer, D., Richard, J., et al. 2018, NatAs, 2, 76

Chary, R., \& Elbaz, D. 2001, ApJ, 556, 562

Combes, F., Rex, M., Rawle, T. D., et al. 2012, A\&A, 538, L4

Connor, T., Donahue, M., Kelson, D. D., et al. 2017, ApJ, 848, 37

Cosens, M., Wright, S. A., Mieda, E., et al. 2018, ApJ, 869, 11

Courteau, S. 1997, AJ, 114, 2402

Cowie, L. L., Hu, E. M., \& Songaila, A. 1995, AJ, 110, 1576

da Cunha, E., Groves, B., Walter, F., et al. 2013, ApJ, 766, 13

Daddi, E., Bournaud, F., Walter, F., et al. 2010, ApJ, 713, 686

Dekel, A., Sari, R., \& Ceverino, D. 2009, ApJ, 703, 785

Dressler, A., Bigelow, B., Hare, T., et al. 2011, PASP, 123, 288

Egami, E., Rex, M., Rawle, T. D., et al. 2010, A\&A, 518, L12

Elmegreen, D. M., Elmegreen, B. G., \& Hirst, A. C. 2004a, ApJL, 604, L21 Elmegreen, D. M., Elmegreen, B. G., \& Sheets, C. M. 2004b, ApJ, 603, 74 Fazio, G. G., Hora, J. L., Allen, L. E., et al. 2004, ApJS, 154, 10 Fernández, X., Gim, H. B., van Gorkom, J. H., et al. 2016, ApJL, 824, L1 Fisher, D. B., Glazebrook, K., Damjanov, I., et al. 2017, MNRAS, 464, 491 Förster Schreiber, N. M., Genzel, R., Bouché, N., et al. 2009, ApJ, 706, 1364 Förster Schreiber, N. M., Shapley, A. E., Erb, D. K., et al. 2011a, ApJ, 731, 65
Förster Schreiber, N. M., Shapley, A. E., Genzel, R., et al. 2011b, ApJ, 739, 45 Frye, B. L., Hurley, M., Bowen, D. V., et al. 2012, ApJ, 754, 17 Geach, J. E., Smail, I., Moran, S. M., et al. 2011, ApJL, 730, L19 Genzel, R., Newman, S., Jones, T., et al. 2011, ApJ, 733, 101 Gómez, P. L., Valkonen, L. E., Romer, A. K., et al. 2012, AJ, 144, 79 Greve, T. R., Vieira, J. D., Weiß, A., et al. 2012, ApJ, 756, 101 Griffin, M. J., Abergel, A., Abreu, A., et al. 2010, A\&A, 518, L3 Gruen, D., Brimioulle, F., Seitz, S., et al. 2013, MNRAS, 432, 1455 Guo, Y., Ferguson, H. C., Bell, E. F., et al. 2015, ApJ, 800, 39 Guo, Y., Giavalisco, M., Ferguson, H. C., Cassata, P., \& Koekemoer, A. M. 2012, ApJ, 757, 120

Henry, R. B. C., \& Worthey, G. 1999, PASP, 111, 919

Hildebrand, R. H. 1983, QJRAS, 24, 267

Hodge, J. A., Swinbank, A. M., Simpson, J. M., et al. 2016, ApJ, 833, 103 Holmberg, E. 1958, MeLuS, 136, 1

Holtzman, J. A., Faber, S. M., Shaya, E. J., et al. 1992, AJ, 103, 691 Houck, J. R., Roellig, T. L., van Cleve, J., et al. 2004, ApJS, 154, 18 Johnson, T. L., Rigby, J. R., Sharon, K., et al. 2017a, ApJL, 843, L21 Johnson, T. L., Sharon, K., Gladders, M. D., et al. 2017b, ApJ, 843, 78 Jones, T., Ellis, R., Jullo, E., \& Richard, J. 2010a, ApJL, 725, L176 Jones, T., Ellis, R. S., Richard, J., \& Jullo, E. 2013, ApJ, 765, 48 Jones, T. A., Swinbank, A. M., Ellis, R. S., Richard, J., \& Stark, D. P. 2010b, MNRAS, 404, 1247

Jullo, E., \& Kneib, J.-P. 2009, MNRAS, 395, 1319

Jullo, E., Kneib, J.-P., Limousin, M., et al. 2007, NJPh, 9, 447

Karman, W., Caputi, K. I., Caminha, G. B., et al. 2017, A\&A, 599, A28

Karman, W., Caputi, K. I., Grillo, C., et al. 2015, A\&A, 574, A11

Kauffmann, G., Heckman, T. M., Tremonti, C., et al. 2003, MNRAS, 346, 1055 Kelson, D. D. 2003, PASP, 115, 688

Kennicutt, R. C., \& Evans, N. J. 2012, ARA\&A, 50, 531

Kennicutt, R. C., Jr. 1998, ARA\&A, 36, 189

Kennicutt, R. C., Jr., Armus, L., Bendo, G., et al. 2003, PASP, 115, 928

Kewley, L. J., \& Ellison, S. L. 2008, ApJ, 681, 1183

Kewley, L. J., Heisler, C. A., Dopita, M. A., \& Lumsden, S. 2001, ApJS, 132,37

Kneib, J.-P., Ellis, R. S., Smail, I., Couch, W. J., \& Sharples, R. M. 1996, ApJ, 471,643

Krist, J. E., Hook, R. N., \& Stoehr, F. 2011, Proc. SPIE, 8127, 81270J

Kruegel, E., \& Siebenmorgen, R. 1994, A\&A, 288, 929

Kümmel, M., Walsh, J. R., Pirzkal, N., Kuntschner, H., \& Pasquali, A. 2009, PASP, 121, 59

Leroy, A. K., Bolatto, A., Gordon, K., et al. 2011, ApJ, 737, 12

Livermore, R. C., Jones, T., Richard, J., et al. 2012, MNRAS, 427, 688

Livermore, R. C., Jones, T. A., Richard, J., et al. 2015, MNRAS, 450, 1812

Madau, P., Pozzetti, L., \& Dickinson, M. 1998, ApJ, 498, 106

Magdis, G. E., Daddi, E., Sargent, M., et al. 2012, ApJL, 758, L9

Mainali, R., Kollmeier, J. A., Stark, D. P., et al. 2017, ApJL, 836, L14

Maughan, B. J., Jones, C., Forman, W., \& Van Speybroeck, L. 2008, ApJS, 174,117

McLeod, B., Fabricant, D., Nystrom, G., et al. 2012, PASP, 124, 1318

Meurer, G. R., Heckman, T. M., Leitherer, C., et al. 1995, AJ, 110, 2665

Mieda, E., Wright, S. A., Larkin, J. E., et al. 2016, ApJ, 831, 78

Mihos, J. C., \& Hernquist, L. 1996, ApJ, 464, 641

Mirabel, I. F., \& Sanders, D. B. 1989, ApJL, 340, L53

Monna, A., Seitz, S., Greisel, N., et al. 2014, MNRAS, 438, 1417

Monreal-Ibero, A., Colina, L., Arribas, S., \& García-Marín, M. 2007, A\&A, 472,421

Moustakas, J., Kennicutt, R. C., Jr., Tremonti, C. A., et al. 2010, ApJS, 190, 233 Noeske, K. G., Weiner, B. J., Faber, S. M., et al. 2007, ApJL, 660, L43

Papadopoulos, P. P., Röttgering, H. J. A., van der Werf, P. P., et al. 2000, ApJ, 528, 626

Peng, C. Y., Ho, L. C., Impey, C. D., \& Rix, H.-W. 2002, AJ, 124, 266

Peng, C. Y., Ho, L. C., Impey, C. D., \& Rix, H.-W. 2010, AJ, 139, 2097

Pérez-González, P. G., Cava, A., Barro, G., et al. 2013, ApJ, 762, 46

Pérez-González, P. G., Rieke, G. H., Villar, V., et al. 2008, ApJ, 675, 234

Pettini, M., \& Pagel, B. E. J. 2004, MNRAS, 348, L59

Piazzo, L., Calzoletti, L., Faustini, F., et al. 2015, MNRAS, 447, 1471

Pilbratt, G. L., Riedinger, J. R., Passvogel, T., et al. 2010, A\&A, 518, L1

Poglitsch, A., Waelkens, C., Geis, N., et al. 2010, A\&A, 518, L2

Postman, M., Coe, D., Benítez, N., et al. 2012, ApJS, 199, 25

Rawle, T. D., Altieri, B., Egami, E., et al. 2016, MNRAS, 459, 1626

Rawle, T. D., Edge, A. C., Egami, E., et al. 2012, ApJ, 747, 29

Rawle, T. D., Egami, E., Bussmann, R. S., et al. 2014, ApJ, 783, 59

Relaño, M., \& Kennicutt, R. C., Jr. 2009, ApJ, 699, 1125

Rex, M., Rawle, T. D., Egami, E., et al. 2010, A\&A, 518, L13

Richard, J., Jauzac, M., Limousin, M., et al. 2014, MNRAS, 444, 268 
Rieke, G. H., Alonso-Herrero, A., Weiner, B. J., et al. 2009, ApJ, 692, 556

Rieke, G. H., Young, E. T., Engelbracht, C. W., et al. 2004, ApJS, 154, 25

Rigby, J. R., Johnson, T. L., Sharon, K., et al. 2017, ApJ, 843, 79

Rigby, J. R., \& Rieke, G. H. 2004, ApJ, 606, 237

Rodighiero, G., Daddi, E., Baronchelli, I., et al. 2011, ApJL, 739, L40

Rupke, D. S. N., Kewley, L. J., \& Chien, L.-H. 2010, ApJ, 723, 1255

Sanders, D. B., \& Mirabel, I. F. 1996, ARA\&A, 34, 749

Sandstrom, K. M., Leroy, A. K., Walter, F., et al. 2013, ApJ, 777, 5

Santini, P., Maiolino, R., Magnelli, B., et al. 2010, A\&A, 518, L154

Schmidt, K. B., Treu, T., Brammer, G. B., et al. 2014, ApJL, 782, L36

Schweizer, F. 1987, in Nearly Normal Galaxies: From the Planck Time to the Present, ed. S. M. Faber (New York: Springer), 18

Siringo, G., Kreysa, E., Kovács, A., et al. 2009, A\&A, 497, 945

Smith, J. D. T., Draine, B. T., Dale, D. A., et al. 2007, ApJ, 656, 770

Sofue, Y., Tutui, Y., Honma, M., et al. 1999, ApJ, 523, 136

Swinbank, A. M., Webb, T. M., Richard, J., et al. 2009, MNRAS, 400, 1121

Thornley, M. D., Schreiber, N. M. F., Lutz, D., et al. 2000, ApJ, 539, 641

Tody, D. 1986, Proc. SPIE, 627, 733
Tody, D. 1993, in ASP Conf. Ser. 52, Astronomical Data Analysis Software and Systems II, ed. R. J. Hanisch, R. J. V. Brissenden, \& J. Barnes (San Francisco, CA: ASP), 173

Treu, T., Schmidt, K. B., Brammer, G. B., et al. 2015, ApJ, 812, 114

Werner, M. W., Roellig, T. L., Low, F. J., et al. 2004, ApJS, 154, 1

Whitaker, K. E., van Dokkum, P. G., Brammer, G., \& Franx, M. 2012, ApJL, 754, L29

Whitmore, B. C., Schweizer, F., Leitherer, C., Borne, K., \& Robert, C. 1993, AJ, 106, 1354

Williamson, R., Benson, B. A., High, F. W., et al. 2011, ApJ, 738, 139

Wisnioski, E., Glazebrook, K., Blake, C., et al. 2012, MNRAS, 422, 3339

Wisnioski, E., Glazebrook, K., Blake, C., \& Swinbank, A. M. 2013, MNRAS, 436, 266

Wong, K. C., Ammons, S. M., Keeton, C. R., \& Zabludoff, A. I. 2012, ApJ, 752, 104

Wuyts, E., Rigby, J. R., Gladders, M. D., \& Sharon, K. 2014, ApJ, 781, 61

Yuan, T.-T., Kewley, L. J., Swinbank, A. M., Richard, J., \& Livermore, R. C. 2011, ApJL, 732, L14 